\title{
DENSE SIGMA-COMPACT SUBSETS OF INFINITE-DIMENSIONAL MANIFOLDS $\left({ }^{1}\right)$
}

\author{
T. A. CHAPMAN
}

\begin{abstract}
In this paper four classes of separable metric infinite-dimensional manifolds are studied; those which are locally the countable infinite product of lines, those which are locally open subsets of the Hilbert cube, and those which are locally one of two dense sigma-compact subsets of the Hilbert cube. A number of homeomorphism, product, characterization, and embedding theorems are obtained concerning these manifolds.
\end{abstract}

1. Introduction. Let $s$ denote the countable infinite product of open intervals and regard the Hilbert cube $I^{\infty}$ as the product of the closures of those intervals. A Fréchet manifold (or $F$-manifold) is a separable metric space having an open cover by sets each homeomorphic to an open subset of $s$. This definition is justified since all separable infinite-dimensional Fréchet spaces are known to be homeomorphic to $s$ (see [7] for references). A Hilbert cube manifold (or Q-manifold) is a separable metric space having an open cover by sets each homeomorphic to an open subset of $I^{\infty}$.

Let $X$ be a separable metric space. A closed subset $K$ of $X$ is said to have Property $Z$ in $X$ (more briefly we say that $K$ is a $Z$-set in $X$ ) provided that for any nonnull homotopically trivial open subset $U$ of $X, U \backslash K$ is nonnull and homotopically trivial. Following Anderson [6] we say that a subset $M$ of $X$ has the (finitedimensional) compact absorption property, or (f-d) cap, in $X$ if (1) $M=\bigcup_{n=1}^{\infty} M_{n}$, where each $M_{n}$ is a (finite-dimensional) compact $Z$-set in $X$ such that $M_{n} \subset M_{n+1}$, and (2) for each $\varepsilon>0$, each integer $m>0$, and each (finite-dimensional) compact subset $K$ of $X$, there is an integer $n>0$ and an embedding $h: K \rightarrow M_{n}$ such that $h \mid K \cap M_{m}=$ id and $d(h, \mathrm{id})<\varepsilon$. We use the convention that (f-d) cap represents two alternatives in an obvious way, one being cap and the other being f-d cap. In this sense we say that $M$ is an (f-d) cap-set for $X$ provided that $M$ has the cap (or $\mathrm{f}-\mathrm{d}$ cap) in $X$.

In [6] R. D. Anderson has established the following theorem: Let $X$ be $I^{\infty}$ or $s$ and let $M, N$ be subsets of $X$, both having the (f-d) cap in $X$. Then there is a

Presented to the Society, January 24, 1970 under the title Four classes of separable metric infinite-dimensional manifolds; received by the editors November 24, 1969.

AMS 1969 subject classifications. Primary 5755; Secondary 5701, 5705.

Key words and phrases. Fréchet manifold, Hilbert cube manifold, compact absorption property, Property $Z$, infinite deficiency, finite-dimensional compact absorption property.

(1) The results of this paper have been submitted as the author's dissertation at Louisiana State University. 
homeomorphism of $X$ onto itself taking $M$ onto $N$. We remark that Bessaga and Pełczyński [12] and Torunczyk [17] have also established this result by use of a device resembling the (f-d) cap.

The question of the equivalence of sets which have the (f-d) cap in an $F$-manifold was raised at a conference on infinite-dimensional topology held at Cornell University, January 5-7, 1969, and it appears as Problem 29 in the report of that meeting, Problems in the topology of infinite-dimensional manifolds. In $\S 6$ we give an affirmative answer to this question.

We will regard the Hilbert cube as a canonical compactification of $s$ in which $I^{\infty}=\prod_{i=1}^{\infty} I_{i}$ and $s=\prod_{i=1}^{\infty} I_{i}^{0}$, where for each $i>0$ we have $I_{i}=[-1,1]$ and $I_{i}^{0}=(-1,1)$. The definition of the (f-d) cap characterizes two different types of subsets of $I^{\infty}$. Let $\sigma$ denote the set of all points in $s$ having at most finitely many nonzero coordinates and let $\Sigma$ denote the set of all points in $s$ having at most finitely many coordinates not in $\left[-\frac{1}{2}, \frac{1}{2}\right]$.

We remark that in real Hilbert space $l^{2}$ there are canonical versions of $\sigma$ and $\Sigma$. The space $\sigma$ is homeomorphic to a vector subspace of $l^{2}$, namely, the linear span of the usual orthonormal basis. The space $\Sigma$ is homeomorphic to a vector subspace of $l^{2}$, namely, the linear span of the usual copy of the Hilbert cube there. It is shown in [6] that $\Sigma$ has the cap in $I^{\infty}$ and $\sigma$ has the f-d cap in $I^{\infty}$.

We will be concerned with manifolds modeled on $\Sigma$ and $\sigma$. A $\Sigma$-manifold (or $\sigma$-manifold) is a separable metric space having an open cover by sets each homeomorphic to an open subset of $\Sigma$ (or $\sigma$ ). In this paper we obtain a number of homeomorphism, characterization, and embedding theorems concerning $F, Q, \Sigma$, and $\sigma$-manifolds. We summarize these results in the next section.

The author wishes to thank R. D. Anderson for helpful comments on an earlier version of this paper.

2. Summary of principal results on $F, Q, \Sigma$, and $\sigma$-manifolds. In this section we list some principal results on $F$ and $Q$-manifolds along with the principal results of this paper. The references refer to results of this paper unless other authorship is indicated.

I. Characterization of manifolds by homotopy type.

THEOREM 2.1 (HENDERSON [14]). If $X$ and $Y$ are F-manifolds of the same homotopy type, then they are homeomorphic.

THEOREM 2.2 (COROLLARY 10.2). If $X$ and $Y$ are both $\sigma$-manifolds or both $\Sigma$ manifolds and are of the same homotopy type, then they are homeomorphic.

CONJECTURE 2.1. If $X$ and $Y$ are compact $Q$-manifolds of the same homotopy type, then they are homeomorphic.

II. Open embedding theorems.

THEOREM 2.3 (HENDERSON [14]). If $X$ is an F-manifold, then there is an open embedding $h: X \rightarrow s$. 
Theorem 2.4 (Theorem 10.3). If $X$ is a $\sigma$ (or $\Sigma$ )-manifold, then $X$ can be embedded as an open subset of $\sigma($ or $\Sigma)$.

III. Product theorems.

THEOREM 2.5 (WEST [18]). If $K$ is any countable locally-finite simplicial complex, then $|K| \times s$ is an $F$-manifold and $|K| \times I^{\infty}$ is a $Q$-manifold.

THEOREM 2.6 (COROLlaRY 8.4). If $K$ is any countable locally-finite simplicial complex, then $|K| \times \sigma$ is a $\sigma$-manifold and $|K| \times \Sigma$ is a $\Sigma$-manifold.

THeOREM 2.7 (COROLlARY 10.3). If $X$ is a $\sigma$-manifold and $Y$ is a $\Sigma$-manifold of the same homotopy type, then $X \times I^{\infty}$ and $Y$ are homeomorphic.

IV. Factor theorems.

THEOREM 2.8 (HENDERSON [14]). If $X$ is an F-manifold, then there is a countable locally-finite simplicial complex $K$ such that $X$ and $|K| \times s$ are homeomorphic.

THEOREM 2.9 (COROLlARY 10.4). If $X$ is a $\sigma$-manifold (or $\Sigma$-manifold), then there is a countable locally-finite simplicial complex $K$ such that $X$ is homeomorphic to $|K| \times \sigma($ or $|K| \times \Sigma)$.

CONJECTURE 2.2. If $X$ is any $Q$-manifold, then there is a countable locally-finite simplicial complex $K$ such that $X$ and $|K| \times I^{\infty}$ are homeomorphic.

THEOREM 2.10 (ANDERSON AND Schori [9]). If $X$ is an F-manifold, then $X, X \times s$, and $X \times I^{\infty}$ are all homeomorphic.

THEOREM 2.11 (ANDERSON AND Schori [9]). If $X$ is a $Q$-manifold, then $X$ and $X \times I^{\infty}$ are homeomorphic.

TheOREM 2.12 (TheOREM 8.3 AND TheOREM 10.2). If $X$ is $a \sigma$-manifold and $I^{n}$ is any n-cell, then $X, X \times \sigma$, and $X \times I^{n}$ are all homeomorphic.

Theorem 2.13 (CoRollary 8.5 AND Theorem 10.2). If $X$ is any $\Sigma$-manifold, then $X, X \times \Sigma$, and $X \times I^{\infty}$ are all homeomorphic.

V. Relationships between $F, Q, \Sigma$, and $\sigma$-manifolds.

TheOREM 2.14 (TheOREM 10.2 AND CoROllary 10.1). If $X$ is a $\sigma$ (or $\Sigma$ )-manifold, then $X$ can be embedded as an f-d cap (or cap)-set for an F-manifold and also for a $Q$-manifold.

THEOREM 2.15 (ANDERSON [6]). If $X$ is $I^{\infty}$ or $s$ and $M, N$ are $(f-d)$ cap-sets for $X$, then there is a homeomorphism of $X$ onto itself taking $M$ onto $N$.

TheOREM 2.16 (Lemma 5.4 AND TheOREM 2.15). If $X$ is an $F$ or $Q$-manifold and $M$ is an $f$-d cap (or cap)-set for $X$, then $M$ is a $\sigma$ (or $\Sigma$ )-manifold.

THEOREM 2.17 (THEOREMS 6.1 AND 6.2). If $X$ is an $F$ or $Q$-manifold and $M, N$ are $(f-d)$ cap-sets for $X$, then there is a homeomorphism of $X$ onto itself taking $M$ onto $N$. 
THEOREM 2.18 (ANDERSON [6]). If $M$ is an $(f-d)$ cap-set for $I^{\infty}$, then $I^{\infty} \mid M$ is homeomorphic to $s$.

THEOREM 2.19 (CoRollary 8.3). If $X$ is any $Q$-manifold and $M$ is an $(f-d)$ cap-set for $X$, then $X \backslash M$ is an F-manifold which is of the same homotopy type as $X$.

VI. Subsets and supersets of $F, Q, \Sigma$, and $\sigma$-manifolds. If $X$ is any space and $\mathscr{U}$ is any cover of $X$, then a function $f: X \rightarrow X$ is said to be limited by $\mathscr{U}$ provided that for each $x \in X$, there is a member of $\mathscr{U}$ containing both $x$ and $f(x)$.

A subset $K$ of a space $X$ is said to be strongly negligible provided that given any open cover $\mathscr{U}$ of $X$, there is a homeomorphism of $X$ onto $X \backslash K$ which is limited by $\mathscr{U}$.

THEOREM 2.20(ANDERSON, HENDERSON, AND West [10]). A necessary and sufficient condition that a closed subset $K$ of an F-manifold have Property $\mathrm{Z}$ is that $K$ be strongly negligible.

THEOREM 2.21 (THEOREMS 6.9 AND 10.2). A necessary and sufficient condition that a closed subset $K$ of a $\sigma$ or $\Sigma$-manifold have Property $Z$ is that $K$ be strongly negligible.

THEOREM 2.22 (THEOREM 6.7). Let $M$ be an $(f-d)$ cap-set for an $F$ or $Q$-manifold $X$ and let $K$ be a Z-set in $X$. Then $M \backslash K$ is an $(f-d)$ cap-set for $X$.

THEOREM 2.23 (TheOREM 6.6). Let $M$ be an $(f-d)$ cap-set for an $F$ or $Q$-manifold $X$ and let $K$ be a countable union of (finite-dimensional) compact Z-sets in $X$. Then $M \cup K$ is an $(f-d)$ cap-set for $X$.

VII. Homeomorphism extension theorems. If $\mathscr{U}$ is any cover of a set $X$, then St $(\mathscr{U})$ is defined (as usual) to be the star of $\mathscr{U}$ and for each $n>0$ we define $\operatorname{St}^{n}(\mathscr{U})$ to be the $n$th star of $\mathscr{U}$.

THEOREM 2.24 (ANDERSON AND MCCHAREN [8]). Let $X$ be an F-manifold, let $K_{1}, K_{2}$ be $Z$-sets in $X$, let $\mathscr{U}$ be an open cover of $X$, and let $h$ be a homeomorphism of $K_{1}$ onto $K_{2}$ for which there is a homotopy $H: K_{1} \times I \rightarrow X$ such that for each $x \in K_{1}$, $H(x, 0)=h(x), H(x, 1)=x$, and $H(\{x\} \times I)$ is contained in some member of $\mathscr{U}$. Then $h$ can be extended to a homeomorphism of $X$ onto itself which is limited by $\operatorname{St}^{4}(\mathscr{U})$.

THEOREM 2.25 (THeOREM 11.1). Let $X$ be a $\sigma$ or $\Sigma$-manifold, let $K_{1}, K_{2}$ be Z-sets in $X$, let $\mathscr{U}$ be an open cover of $X$, and let $h$ be a homeomorphism of $K_{1}$ onto $K_{2}$ for which there is a homotopy $H: K_{1} \times I \rightarrow X$ such that for each $x \in K_{1}, H(x, 0)=h(x)$, $H(x, 1)=x$, and $H(\{x\} \times I)$ is contained in some member of $\mathscr{U}$. Then $h$ can be extended to a homeomorphism of $X$ onto itself which is limited by $\mathrm{St}^{28}(\mathscr{U})$.

VIII. Complete extensions of $\Sigma$ and $\sigma$-manifolds.

TheOREM 2.26 (LeMMA 10.1 AND TheOREM 10.2). Let $X$ be a $\sigma$ (or $\Sigma$ )-manifold and let $Y$ be a complete separable metric space containing $X$. Then there is an $F$ manifold $Z$ such that $X \subset Z \subset Y$ and $X$ is an $f$-d cap (or cap)-set for $Z$. 
IX. Infinite deficiency. A subset $K$ of $I^{\infty}$ is said to have infinite deficiency provided that for each of infinitely many different coordinate directions, $K$ projects onto a single interior point of $(-1,1)$.

THEOREM 2.27 (ANDERSON [3]). Let $X$ be $I^{\infty}$ or $s$ and let $K$ be a closed subset of $X$. $A$ necessary and sufficient condition that $K$ be a Z-set in $X$ is that there exists a homeomorphism of $X$ onto itself taking $K$ onto a set having infinite deficiency.

TheOREM 2.28 (CHAPMAN [13]). Let $X$ be an F-manifold and let $K$ be a closed subset of $X . A$ necessary and sufficient condition that $K$ be a Z-set in $X$ is that there exists a homeomorphism $h$ of $X$ onto $X \times s$ such that $\pi_{s} \circ h(K)$ has infinite deficiency.

We remark that a separate proof of Theorem 2.28 is given in Corollary 7.1 of this paper.

TheOREM 2.29 (TheOREM 7.2). Let $X$ be a $Q$-manifold and let $K$ be a closed subset of $X$. A necessary and sufficient condition that $K$ be a Z-set in $X$ is that there exists a homeomorphism $h$ of $X$ onto $X \times I^{\infty}$ such that $\pi_{I}^{\infty} \circ h(K)$ has infinite deficiency.

3. Preliminaries. The metric we use for $I^{\infty}$ and $s$ is given by

$$
d\left(\left(x_{i}\right),\left(y_{i}\right)\right)=\left(\sum_{i=1}^{\infty} 2^{-i}\left(x_{i}-y_{i}\right)^{2}\right)^{1 / 2},
$$

where $\left(x_{i}\right),\left(y_{i}\right) \in I^{\infty}$. Whenever no confusion is possible we will use $d$ to denote the metric of any space under consideration.

Let $Z$ denote the set of positive integers and for each $i \in Z$ let $\tau_{i}$ be the projection of $I^{\infty}$ onto $I_{i}$. For each $\alpha \subset Z$ define $I^{\alpha}=\prod_{i \in \alpha} I_{i}, s^{\alpha}=\prod_{i \in \alpha} I_{i}^{0}$, and let $\tau_{\alpha}$ be the projection of $I^{\infty}$ onto $I^{\alpha}$. For each $i \in Z$ let $W_{i}^{+}=\tau_{i}^{-1}(1), W_{i}^{-}=\tau_{i}^{-1}(-1)$, and $W_{i}=W_{i}^{+} \cup W_{i}^{-}$. We call $W_{i}^{+}$and $W_{i}^{-}$the endslices of $I^{\infty}$ in the $i$-direction.

A subset of $I^{\infty}$ of the form $\prod_{i=1}^{\infty} J_{i}$ is called a basic closed set in $I^{\infty}$ provided that $J_{i}$ is a closed subinterval of $I_{i}$, for each $i>0$, and $J_{i}=I_{i}$ for all but finitely many $i$. The interior in $I^{\infty}$ of a basic closed set is called a basic open set in $I^{\infty}$. A basic open set in $s$ is the intersection of a basic open set in $I^{\infty}$ with $s$. We call $s$ the pseudointerior of $I^{\infty}$ and $B\left(I^{\infty}\right)=I^{\infty} \mid s$ the pseudo-boundary of $I^{\infty}$.

A core is a set $C=\prod_{i=1}^{\infty} J_{i}$, where for each $i>0, J_{i}$ is a closed interval contained in $I_{i}^{0}$. A basic core set structured over a core $C=\prod_{i=1} J_{i}$ is defined as the set of all points $\left(x_{i}\right) \in s$ such that $x_{i} \in J_{i}$, for all but finitely many $i$. We know from [4] that each basic core set is an apparent boundary of $I^{\infty}$ and from [6] that each apparent boundary of $I^{\infty}$ is a cap-set for $I^{\infty}$, where $M \subset I^{\infty}$ is an apparent boundary if there is a homeomorphism $h: I^{\infty} \rightarrow I^{\infty}$ satisfying $h(M)=B\left(I^{\infty}\right)$.

A homeomorphism $h$ of $I^{\infty}$ onto itself is said to be a $\beta^{*}$-homeomorphism provided that $h(s)=s$.

If $\left\{f_{i}\right\}_{i=1}^{\infty}$ is a sequence of homeomorphisms of a space $X$ onto itself for which the sequence $\left\{f_{i} \circ f_{i-1} \circ \cdots \circ f_{1}\right\}$ converges pointwise to a homeomorphism $f$ of $X$ onto itself, then we call $f$ the infinite left product of $\left\{f_{i}\right\}_{i=1}^{\infty}$ and write $f=L \prod_{i=1}^{\infty} f_{i}$. 
We list below four convergence procedures that we will need to insure the existence of an infinite left product of homeomorphisms. The first of these is Lemma 2.1 of [3] and the second is Theorem 2 of [10]. The third and fourth are easy consequences of the apparatus used in [10] to establish the second.

Convergence Procedure A. For each homeomorphism $g$ of a compact metric space $X$ onto itself and each $\varepsilon>0$ let

$$
\eta(g, \varepsilon)=\text { g.l.b. }\{d(g(x), g(y)) \mid d(x, y) \geqq \varepsilon\} .
$$

If $\left\{f_{i}\right\}_{i=1}^{\infty}$ is a sequence of homeomorphisms of $X$ onto itself such that

$$
d\left(f_{i}, \mathrm{id}\right)<\min \left(\left(3^{-i}\right),\left(3^{-i}\right) \cdot \eta\left(f_{i-1} \circ \cdots \circ f_{1}, 2^{-i}\right)\right),
$$

for all $i>1$, then $f=L \prod_{i=1}^{\infty} f_{i}$ exists.

Convergence Procedure B. Let $\mathscr{U}$ be a countable star-finite open cover of any space X. (By star-finite cover we mean a cover such that the closure of each member of the cover intersects the closure of only finitely many other members of the cover.) There exists an ordering $\left\{U_{i}\right\}_{i=1}^{\infty}$ of the elements of $\mathscr{U}$ such that for any sequence $\left\{f_{i}\right\}_{i=1}^{\infty}$ of homeomorphisms of $X$ onto itself, where $f_{i}$ is the identity on $X \backslash U_{i}$ for all $i>0, f=L \prod_{i=1}^{\infty} f_{i}$ exists. Moreover, we can assign a positive integer $n_{i}$ to each $U_{i}$, independent of the choice of such $\left\{f_{i}\right\}_{i=1}^{\infty}$, such that $n_{i} \leqq n_{i+1}$ and

$$
f\left(U_{i}\right)=\left(f_{n_{i}} \circ f_{n_{i}-1} \circ \cdots \circ f_{1}\right)\left(U_{i}\right),
$$

for all $i>0$.

Convergence Procedure C. Let $\mathscr{U}$ be a countable star-finite open cover of any space $X$ and let $\left\{U_{i}\right\}_{i=1}^{\infty},\left\{n_{i}\right\}_{i=1}^{\infty}$ be as in Convergence Procedure B. If $Y$ is any space and $\left\{f_{i}\right\}_{i=1}^{\infty}$ is any sequence of homeomorphisms of $X \times Y$ onto itself such that $f_{i}$ is the identity on $\left(X \backslash U_{i}\right) \times Y$, for all $i>0$, then $f=L \prod_{i=1}^{\infty} f_{i}$ exists. Moreover, we have

$$
f\left(U_{i} \times Y\right)=\left(f_{n_{i}} \circ \cdots \circ f_{1}\right)\left(U_{i} \times Y\right),
$$

for all $i>0$.

Convergence Procedure D. Let $\mathscr{U}$ be a countable star-finite open cover of any space $X$ and let $\left\{U_{i}\right\}_{i=1}^{\infty}$ be as in Convergence Procedure B. If $\left\{f_{i}\right\}_{i=1}^{\infty}$ is any sequence of homeomorphisms of $X$ onto itself such that $f_{1}|X| U_{1}=\mathrm{id}$ and $f_{i+1}|X| f_{i} \circ \cdots \circ f_{1}\left(U_{i+1}\right)$ $=\mathrm{id}$, for each $i>0$, then $f=L \prod_{i=1}^{\infty} f_{i}$ exists.

4. Some basic results concerning (f-d) cap-sets. The following result can be found in [6].

LEMMA 4.1. Let $X$ be a metric space, let $M$ be an $(f-d)$ cap-set for $X$, and let $f$ be a homeomorphism of $X$ onto a metric space $Y$. Then $f(M)$ is an $(f-d)$ cap-set for $Y$.

The next result shows that $(f-d)$ cap-sets in $I^{\infty}$ are "maximal." We will use this result to prove a corresponding property for $F$ and $Q$-manifolds in $\S 6$. We will give no proof, since it is essentially Theorem 1 of [17]. We remark that Lemma 4.2 follows from Theorem II of [4] in the case that $M$ is a cap-set for $I^{\infty}$. 
LEMmA 4.2. Let $M \subset N \subset I^{\infty}$, where $M$ is an $(f-d)$ cap-set for $I^{\infty}$ and $N$ is a countable union of (finite-dimensional) Z-sets in $I^{\infty}$. Then $N$ is an $(f-d)$ cap-set for $I^{\infty}$.

Our next lemma is a generalization of Anderson's results on the equivalence of (f-d) cap-sets in $s$ and $I^{\infty}[6]$. We only sketch the proof.

LEMMA 4.3. Let $M$ and $N$ be $(f-d)$ cap-sets for $I^{\infty}$, let $K \subset I^{\infty} \mid(M \cup N)$ be a $Z$-set in $I^{\infty}$, and let $\varepsilon>0$ be given. Then there is a homeomorphism $h$ of $I^{\infty}$ onto itself such that $h(M)=N, h \mid K=\mathrm{id}$, and $d(h$, id $)<\varepsilon$. Moreover, if $M \cup N \subset s$, then we can additionally require that $h$ be a $\beta^{*}$-homeomorphism.

Proof. We only treat the case in which $M \cup N \subset s$ to obtain the $\beta^{*}$-homeomorphism $h$. Write $M=\bigcup_{n=1}^{\infty} M_{n}$ and $N=\bigcup_{n=1}^{\infty} N_{n}$ so as to satisfy the definition. It is clear that there is an integer $n_{1}>0$ and an embedding $f_{1}: M_{1} \rightarrow N_{n_{1}}$ such that $d\left(f_{1}, \mathrm{id}\right)<\varepsilon / 2$. Using Lemma 3.1 of [13], we can extend $f_{1}$ to a $\beta^{*}$-homeomorphism $h_{1}$ such that $h_{1} \mid K=\mathrm{id}$ and $d\left(h_{1}, \mathrm{id}\right)<\varepsilon / 2$.

Using Convergence Procedure A let $\delta=\min \left(\left(3^{-2}\right),\left(3^{-2}\right) \eta\left(h_{1}, 2^{-2}\right)\right)$ and use Lemma 4.1 to get an integer $n_{2}>1$ and an embedding $f_{2}: N_{n_{1}} \rightarrow h_{1}\left(M_{n_{2}}\right)$ such that $f_{2} \mid h_{1}\left(M_{1}\right)=$ id and $d\left(f_{2}\right.$, id $)<\min (\varepsilon / 4, \delta)$. Then we can extend $f_{2}$ to a $\beta^{*}$-homeomorphism $g_{2}$ such that $g_{2} \mid\left(K \cup W_{1} \cup h_{1}\left(W_{1}\right)\right)=\mathrm{id}$ and $d\left(g_{2}, \mathrm{id}\right)<\min (\varepsilon / 4, \delta)$. We then put $h_{2}=g_{2}^{-1}$ to complete the second stage of the construction.

It is clear that by induction we can continue this process to obtain a sequence $\left\{h_{i}\right\}_{i=1}^{\infty}$ of $\beta^{*}$-homeomorphisms and a sequence $\left\{n_{i}\right\}_{i=0}^{\infty}$ of integers (where $n_{0}=1$ ) such that the following conditions are met.

(1) $n_{0}<n_{2}<n_{4}<\cdots$ and $n_{1}<n_{3}<n_{5}<\cdots$,

(2) for each $i>1, h_{i}$ is the identity on $K \cup\left(\bigcup_{j=1}^{i-1} W_{j}\right) \cup\left(h_{i-1} \circ \cdots \circ h_{1}\right)\left(\bigcup_{j=1}^{i-1} W_{j}\right)$,

(3) $\left(h_{i} \circ \cdots \circ h_{1}\right)\left(M_{n_{i-1}}\right) \subset N_{n_{i}}$, for $i$ odd,

(4) $\left(h_{i} \circ \cdots \circ h_{1}\right)\left(M_{n_{i}}\right) \supset N_{n_{i-1}}$, for $i$ even,

(5) $h_{i}$ is the identity on $N_{n_{i-2}}$, for $i \geqq 3$ and odd,

(6) $h_{i}$ is the identity on $h_{i-1} \circ \cdots \circ h_{1}\left(M_{n_{i-2}}\right)$, for $i$ even,

(7) $d\left(h_{1}\right.$, id $)<\varepsilon / 2$ and $d\left(h_{i}\right.$, id $)<\min \left(\varepsilon / 2^{i}, 3^{-i},\left(3^{-i}\right) \eta\left(h_{i-1} \circ \ldots \circ h_{1}, 2^{-i}\right)\right)$, for $i>1$.

Using Convergence Procedure A we find that $h=L \prod_{i=1}^{\infty} h_{i}$ is a $\beta^{*}$-homeomorphism which satisfies the required properties.

A special case of the next lemma is known in a more general setting (see Corollary 2 of [17]).

Lemma 4.4. Let $M$ be an $(f-d)$ cap-set for $I^{\infty}$ and let $K$ be a Z-set in $I^{\infty}$. Then $M \backslash K$ is an $(f-d)$ cap-set for $I^{\infty}$.

Proof. Let $M=\bigcup_{n=1}^{\infty} M_{n}$ be as in the definition and for each integer $n>0$ let $N_{n}=M_{n} \cap\left\{x \in I^{\infty} \mid d(x, K) \geqq 1 / n\right\}$. It is then clear that $M \backslash K=\bigcup_{n=1}^{\infty} N_{n}$. It follows from Theorems 8.1 and 9.1 of [3] that Property $\mathrm{Z}$ and infinite deficiency are equivalent in $I^{\infty}$ modulo ambient homeomorphisms. This implies that each $N_{n}$ is a $Z$-set in $I^{\infty}$. 
Now let $A$ be a (finite-dimensional) compact subset of $I^{\infty}$, let $m>0$ be an integer, and let $\varepsilon>0$ be given. It follows from Theorem II of [4] that there is a cap-set $M^{\prime}$ for $I^{\infty}$ in $I^{\infty} \mid K$. Let $M^{\prime}=\bigcup_{n=1}^{\infty} M_{n}^{\prime}$ be a representation as in the definition. Without loss of generality we may assume that $A$ is a $Z$-set. Let $A \backslash N_{m}=\bigcup_{n=1}^{\infty} C_{n}$, where each $C_{n}$ is a compact set satisfying $C_{n} \subset C_{n+1}$. Let $n_{1}>0$ be chosen large enough so that there is an embedding $f_{1}: C_{1} \rightarrow M_{n_{1}}^{\prime}$ which satisfies $f_{1}\left(C_{1}\right) \cap N_{m}=\varnothing$ and $d\left(f_{1}\right.$, id $)<\varepsilon / 4$. Using Lemma 3.1 of [13] we can extend $f_{1}$ to a homeomorphism $f_{1}^{\prime}: I^{\infty} \rightarrow I^{\infty}$ which satisfies $f_{1}^{\prime} \mid N_{m}=$ id and $d\left(f_{1}^{\prime}\right.$, id $)<\varepsilon / 4$.

Let $\varepsilon^{\prime}=\min \left(\varepsilon / 8,3^{-2},\left(3^{-2}\right) \eta\left(f_{1}^{\prime}, 2^{-2}\right)\right)$ and choose $n_{2}>n_{1}$ large enough so that there is an embedding $f_{2}: f_{1}^{\prime}\left(C_{2}\right) \rightarrow M_{n_{2}}^{\prime}$ which satisfies $f_{2}\left(f_{1}^{\prime}\left(C_{2}\right)\right) \cap N_{m}=\varnothing$, $f_{2} \mid f_{1}^{\prime}\left(C_{1}\right)=\mathrm{id}$, and $d\left(f_{2}, \mathrm{id}\right)<\varepsilon^{\prime}$. Once more use Lemma 3.1 of [13] to extend $f_{2}$ to a homeomorphism $f_{2}^{\prime}: I^{\infty} \rightarrow I^{\infty}$ which satisfies $f_{2}^{\prime} \mid N_{m}=$ id and $d\left(f_{2}^{\prime}\right.$, id $)<\varepsilon^{\prime}$.

Using Convergence Procedure A we can continue this process in an obvious fashion to obtain a sequence $\left\{f_{i}^{\prime}\right\}_{i=1}$ of homeomorphisms of $I^{\infty}$ onto itself for which $f=L \prod_{i=1} f_{i}^{\prime}$ exists and satisfies $f\left(A \backslash N_{m}\right) \subset M^{\prime}, f \mid N_{m}=\mathrm{id}$, and $d(f$, id $)<\varepsilon / 2$.

Let $\delta=d(f(A), K)$, which is a positive number, and let $g: f(A) \rightarrow M_{n}$ be an embedding, for some $n>0$, such that $g \mid A \cap N_{m}=\mathrm{id}$ and $d(g, \mathrm{id})<\min (\varepsilon / 2, \delta)$. Then $g \circ f: A \rightarrow M_{n}$ is an embedding for which $g \circ f(A) \cap K=\varnothing$ and $d(g \circ f$, id $)<\varepsilon$. It is clear that there is an $n^{\prime} \geqq n$ such that $g \circ f(A) \subset N_{n^{\prime}}$, which completes the verification of the definition.

Corollary 4.1. Let $M$ be an $(f-d)$ cap-set for $s$ and let $K$ be a Z-set in s. Then $M \backslash K$ is an $(f-d)$ cap-set for $s$.

Proof. We know from Theorem 8.2 of [3] that $\bar{K}$, the closure of $K$ in $I^{\infty}$, is a $Z$-set in $I^{\infty}$. Now any (f-d) cap-set for $s$ is an (f-d) cap-set for $I^{\infty}$, and any subset of $s$ which is an (f-d) cap-set for $I^{\infty}$ is an (f-d) cap-set for $s$. Then $M$ is an (f-d) cap-set for $I^{\infty}$ and from Lemma 4.4 we find that $M \backslash \bar{K}=M \backslash K$ is an (f-d) cap-set for $s$.

5. Property $\mathbf{Z}$ in manifolds. In this section we prove some results concerning Property $\mathrm{Z}$ in manifolds that will be useful in later sections. We also exhibit a technique (Theorem 5.1 ) by which we can relate $F$ and $Q$-manifolds.

In Lemma 1 of [10] some techniques are given for establishing Property $Z$ in normal, locally homotopically trivial spaces. Lemma 5.1 is essentially a restatement of this result and Lemma 5.2 is a consequence of the proof that is given there.

LEMMA 5.1. Let $X$ be normal and locally homotopically trivial. If $A$ is a closed subset of $X$ such that each point of $A$ lies in an open set $U$ for which $A \cap U$ has Property $\mathrm{Z}$ in $U$, then $A$ has Property $\mathrm{Z}$ in $X$.

LEMMA 5.2. Let $X$ be normal and locally homotopically trivial and let $A$ be a closed subset of $X$. If, for each point $p \in A$ and each open set $U$ containing $p$, there is a homotopically trivial open set $V$ containing $p$ such that $V \subset U, V \backslash A \neq \varnothing$, and $V \backslash A$ is homotopically trivial, then $A$ has Property $\mathrm{Z}$ in $X$. 
In Lemma 2 of [10] it is shown that if $F$ is any $Z$-set in an $F$-manifold $X$, then any closed subset of $F$ is also a $Z$-set in $X$. Lemma 5.3 establishes a similar property for $Q$-manifolds. We remark that for $F$-manifolds all compact subsets are $Z$-sets. This follows from Theorem 2.1 and the fact that any compact subset of $s$ is a $Z$-set (see Theorem 3.5 of [2] and Theorem 9.1 of [3]).

LEMMA 5.3. Let $F$ be a $Z$-set in any $Q$-manifold $X$. If $A$ is any closed subset of $F$, then $A$ is also a Z-set in $X$.

Proof. Using Lemma 5.1 let $U$ be any open subset of $X$ which is homeomorphic to a basic open subset of $I^{\infty}$. Then there is a homeomorphism $h$ of $U$ onto $I^{\infty} \mid W$, where $W$ is a finite union of endslices of $I^{\infty}$. Since Property $Z$ obviously carries over to open subsets we find that $h(F \cap U)$ is a $Z$-set in $I^{\infty} \mid W$.

Define $K=h(F \cap U) \cup W$, which is a compact subset of $I^{\infty}$. Let $V$ be any nonnull, homotopically trivial open subset of $I^{\infty}$ and note that $V \backslash K=$ $(V \backslash W) \backslash h(F \cap U)$. Since $W$ obviously has Property $\mathrm{Z}$ in $I^{\infty}$ it follows that $V \backslash W$ is a nonnull, homotopically trivial open subset of $I^{\infty} \mid W$, hence $V \backslash K$ is a nonnull, homotopically trivial open set. This means that $K$ is a $Z$-set in $I^{\infty}$.

Using Theorem 8.1 of [3] there is a homeomorphism $g$ of $I^{\infty}$ onto itself such that $g(K)$ has infinite deficiency. Using techniques similar to those of Theorem 9.1 of [3] it now easily follows that $g \circ h(A \cap U)$ is a $Z$-set in $g \circ h(U)$.

The following result was pointed out to the author by William Barit.

LEMMA 5.4. Let $X$ be an $F$ or $Q$-manifold and let $M$ be an $(f-d)$ cap-set for $X$. If $U$ is an open subset of $X$, then $M \cap U$ is an $(f-d)$ cap-set for $U$.

Proof. Let $M=\bigcup_{n=1}^{\infty} M_{n}$ be a representation as given in the definition and for each $n>0$, let $A_{n}=\{x \in X \mid d(x, X \mid U) \geqq 1 / n\}$. If we put $N=M \cap U$ and $N_{n}=M_{n} \cap A_{n}$, for each $n>0$, then $N=\bigcup_{n=1}^{\infty} N_{n}$. It follows from Lemma 5.3 and the comments preceding Lemma 5.3 that each $N_{n}$ is a $Z$-set in $U$. Then $N=\bigcup_{n=1}^{\infty} N_{n}$ is clearly a representation which satisfies the definition for $N$ to be an (f-d) cap-set for $U$.

Since (f-d) cap-sets for $s$ exist, we can use Theorem 2.1 and Lemma 5.4 to prove the existence of $(\mathrm{f}-\mathrm{d})$ cap-sets for $F$-manifolds. In Lemma 5.6 we prove that capsets for $Q$-manifolds exist. The existence of $\mathrm{f}$-d cap-sets for $Q$-manifolds will be established in $\$ 6$. We will first need a preliminary result.

Lemma 5.5. Let $X, Y$ be $Q$-manifolds and let $A$ be a Z-set in $X$. Then $A \times Y$ is a $Z$-set in $X \times Y$.

Proof. Using Lemma 5.2 let $U$ be a nonnull, homotopically trivial open subset of $X$ and let $V$ be a nonnull, homotopically trivial open subset of $Y$. All we have to do is prove that $(U \times V) \mid(A \times Y)$ is nonnull and homotopically trivial. But $(U \times V) \mid(A \times Y)=(U \backslash A) \times V$ is clearly nonnull and homotopically trivial.

Lemma 5.6. If $X$ is any $Q$-manifold, then $X$ has a cap-set. 
Proof. Using Theorem 2.11 we know that there is a homeomorphism of $X$ onto $X \times I^{\infty}$. It will then suffice to prove that $X \times \Sigma$ has the cap in $X \times I^{\infty}$. Since $\Sigma$ has the cap in $I^{\infty}$ we can write $\Sigma=\bigcup_{n=1}^{\infty} M_{n}$ so as to satisfy the definition. Write $X=\bigcup_{n=1}^{\infty} X_{n}$, where each $X_{n}$ is a compact subset of $X$ lying in the interior of $X_{n+1}$. Then $X \times \Sigma=\bigcup_{n=1}^{\infty}\left(X_{n} \times M_{n}\right)$ which we now show to satisfy the definition of a cap-set for $X \times I^{\infty}$.

It follows from Lemmas 5.3 and 5.5 that each $X_{n} \times M_{n}$ is a $Z$-set in $X \times I^{\infty}$. Now let $m>0$ be an integer, let $K$ be a compact subset of $X \times I^{\infty}$, and let $\varepsilon>0$ be given. Since $\pi_{I}{ }^{\infty}(K)$ is a compact subset of $I^{\infty}$ there is an integer $n_{1}>0$ and an embedding $h_{1}: \pi_{I}^{\infty}(K) \rightarrow M_{n_{1}}$ such that $h_{1} \mid \pi_{I}^{\infty}(K) \cap M_{m}=$ id and $d\left(h_{1}\right.$, id $)<\varepsilon$. It is clear that there is an integer $n_{2}>0$ such that $\pi_{X}(K) \subset X_{n_{2}}$. Put $n=\max \left(n_{1}, n_{2}\right)$ and we obviously have an embedding $h$ of $K$ into $X_{n} \times M_{n}$ such that $h \mid K \cap\left(X_{m} \times M_{m}\right)=$ id and $d(h, \mathrm{id})<\varepsilon$.

With the existence of cap-sets in $Q$-manifolds established we can now prove a result which relates $F$ and $Q$-manifolds.

THEOREM 5.1. If $X$ is any F-manifold, then $X$ can be regarded as a subset of a $Q$-manifold $Y$ such that $Y \backslash X$ is a cap-set for $Y$. On the other hand, if $Y$ is any $Q$-manifold, then there is an $F$-manifold $X \subset Y$ such that $Y \backslash X$ is a cap-set for $Y$.

Proof. If $X$ is any $F$-manifold, then Theorem 2.1 gives an open embedding $h: X \rightarrow s$. Let $Y$ be an open subset of $I^{\infty}$ such that $Y \cap s=h(X)$. Then $Y$ is obviously a $Q$-manifold and it follows from Lemma 5.4 , and the fact that $B\left(I^{\infty}\right)$ is a cap-set for $I^{\infty}$, that $Y \backslash h(X)$ is a cap-set for $Y$.

If $Y$ is any $Q$-manifold, it follows from Lemma 5.6 that there is a cap-set $M$ for $Y$. To show that $Y \backslash M$ is an $F$-manifold let $U$ be any open subset of $Y$ which is homeomorphic to a basic open subset of $I^{\infty}$. Let $h: U \rightarrow I^{\infty}$ be an embedding such that $I^{\infty} \mid h(U)=W$, where $W$ is a finite union of endslices of $I^{\infty}$. It is obvious that $h(U \cap M)$ is a cap-set for $I^{\infty}$ and we know that $\Sigma$ is a cap-set for $I^{\infty}$. Using Lemma 4.3 there is a homeomorphism $g$ of $I^{\infty}$ onto itself such that $g(\Sigma)=h(U \cap M)$ and $g \mid W=$ id. This means that $h(U \mid M)$ is homeomorphic $I^{\infty} \mid(\Sigma \cup W)$. But Theorem 2.18 implies that $I^{\infty} \mid(\Sigma \cup W)$ is homeomorphic to $s$.

Corollary 5.1. Let $Y$ be any $Q$-manifold and let $M$ be an $(f-d)$ cap-set for $Y$. Then $Y \backslash M$ is an F-manifold.

Proof. For $M$ a cap-set the proof is given above. For $M$ an $\mathrm{f}-\mathrm{d}$ cap-set we use the outline given above.

In Theorem 8.2 of [3] it is shown that if $F$ is a $Z$-set in $s$, then $\bar{F}$ (closure taken in $I^{\infty}$ ) is a $Z$-set in $I^{\infty}$. The next result generalizes this to $Q$-manifolds.

LEMMA 5.7. Let $X$ be a $Q$-manifold and let $M$ be a cap-set for $X$. If $F$ is a Z-set in $X \backslash M$, then $\bar{F}$ (closure taken in $X$ ) is a Z-set in $X$.

Proof. Using Lemma 5.1 let $U$ be an open subset of $X$ and let $h: U \rightarrow I^{\infty}$ be an embedding such that $I^{\infty} \mid h(U)=W$, where $W$ is a finite union of endslices of $I^{\infty}$. 
Using the proof of Theorem 5.1 we can additionally require that $h(U \cap M)$ $=B\left(I^{\infty}\right) \backslash W$. Then $h(U \backslash M)=s$ and hence $K=h(F \cap U)$ is a $Z$-set in $s$. This means that $\bar{K}$ is a $Z$-set in $I^{\infty}$. Then $h(\bar{F} \cap U)=\bar{K} \cap h(U)$ is a $Z$-set in $h(U)$, which is what we needed.

The next result will be used in proving an open embedding theorem for $\sigma$ and $\Sigma$-manifolds.

LEMMA 5.8. Let $X$ be an F-manifold, let $M$ be an $(f-d)$ cap-set for $X$, and let $F$ be a closed subset of $X$ contained in $X \backslash M$. Then $F$ is a Z-set in $X$.

Proof. Using Lemma 5.1 it is sufficient to prove that for any open subset $U$ of $X$ which is homeomorphic to $s, U \cap F$ is a $Z$-set in $U$. Thus, our problem is reduced to the following: if $N$ is an (f-d) cap-set for $s$ and $K$ is a closed subset of $s$ contained in $s \backslash N$, then $K$ is a $Z$-set in $s$.

To show that $K$ has Property $\mathrm{Z}$ in $s$ let $V$ be a nonnull, homotopically trivial open subset of $s$. Let $f: S^{n-1} \rightarrow V \backslash K$ be continuous and let $g: B^{n} \rightarrow V$ be a continuous extension of $f$, where $B^{n}$ is an $n$-ball and $S^{n-1}$ is the boundary of $B^{n}$. If we think of a canonical version of $N$ (i.e. $N=\sigma$ or $N=\Sigma$ ) then it is clear that there is a continuous function $h: g\left(B^{n}\right) \rightarrow s$ such that $h\left(g\left(B^{n}\right) \backslash g\left(S^{n-1}\right)\right) \subset N, h \mid g\left(S^{n-1}\right)=\mathrm{id}$, and $d(h$, id $)<\varepsilon$, where $\varepsilon=d\left(g\left(B^{n}\right), s \backslash V\right)$. Note that $h\left(g\left(B^{n}\right)\right) \cap K=\varnothing, h\left(g\left(B^{n}\right)\right) \subset V$, and $h \circ g$ is a continuous extension of $f$. This proves that $V \backslash K$ is homotopically trivial. Since $V \cap N \neq \varnothing$ we have $V \backslash K \neq \varnothing$.

The following two results will be used in the last section of the paper.

LEMMA 5.9. Let $X$ be any F-manifold, let $F$ be a Z-set in $X$, and for each $n>0$ let $F_{n}$ be a Z-set in $X$. Then $F \cap\left(X \backslash \bigcup_{n=1}^{\infty} F_{n}\right)$ is a Z-set in $X \backslash \bigcup_{n=1}^{\infty} F_{n}$.

Proof. Using Lemma 5.2 let $U$ be any nonnull, homotopically trivial open subset of $X$. Then $U \cap\left(X \mid \bigcup_{n=1}^{\infty} F_{n}\right)=U \backslash \bigcup_{n=1}^{\infty}\left(F_{n} \cap U\right)$, and using the negligibility result of [4] we know that $U \backslash \bigcup_{n=1}^{\infty}\left(F_{n} \cap U\right)$ is homeomorphic to $U$. Thus $U \cap\left(X \backslash \bigcup_{n=1}^{\infty} F_{n}\right)$ is homotopically trivial. We then have $U \cap\left(X \backslash \bigcup_{n=1}^{\infty} F_{n}\right) \mid F$ nonnull and homotopically trivial by the same argument. Lemma 5.2 then implies that $F \cap\left(X \backslash \bigcup_{n=1}^{\infty} F_{n}\right)$ is a $Z$-set in $X \backslash \bigcup_{n=1}^{\infty} F_{n}$.

LEMMA 5.10. Let $X$ be an F-manifold, let $M \subset X$ be an $(f-d)$ cap-set for $X$, and let $F$ be a Z-set in $M$. Then $\bar{F}($ closure in $X)$ is a Z-set in $X$.

Proof. Using Lemma 5.1 it is clear that our problem reduces to the following: If $N$ is an (f-d) cap-set for $s$ and $K$ is a $Z$-set in $N$, then $\bar{K}$ (closure in $s$ ) is a $Z$-set in $s$.

Let $U$ be a nonnull, homotopically trivial open subset of $s$, let $f: S^{n-1} \rightarrow U \backslash \bar{K}$ be a continuous function, and let $g: B^{n} \rightarrow U$ be a continuous extension of $f$. Using the idea contained in the proof of Lemma 5.8 there is a continuous function $h: B^{n} \rightarrow s$ such that $h\left(g\left(B^{n}\right) \mid g\left(S^{n-1}\right)\right) \subset N, h \mid g\left(S^{n-1}\right)=\mathrm{id}$, and $h \circ g\left(B^{n}\right) \subset U$. This argument has already shown that $U \cap N$ is homotopically trivial. 
It is clear that there is an $r, 0<r<1$, such that $h \circ g\left(\left\{x \in B^{n} \mid r \leqq\|x\| \leqq 1\right\}\right)$ $\cap \bar{K}=\varnothing$. Put $S_{r}^{n-1}=\left\{x \in B^{n} \mid\|x\|=r\right\}$ and use the fact that $K$ has Property $\mathrm{Z}$ in $N$ to extend the map $h \circ g\left|S_{r}^{n-1}: S_{r}^{n-1} \rightarrow(U \cap N)\right| K$ to a continuous function $\varphi: B_{r}^{n}$ $\rightarrow(U \cap N) \mid K$, where $B_{r}^{n}=\left\{x \in B^{n} \mid\|x\| \leqq r\right\}$. Piecing these together we then have a continuous function $\psi: B^{n} \rightarrow U \backslash \bar{K}$ which extends $f$.

6. Properties of (f-d) cap-sets in $F$ and $Q$-manifolds. The main results of this section are Theorems 6.1 and 6.2, in which we establish the equivalence of (f-d) cap-sets in $F$ and $Q$-manifolds. We also use similar techniques to obtain a number of other results.

We first establish the equivalence of (f-d) cap-sets in $F$-manifolds.

THEOREM 6.1. Let $X$ be an F-manifold, let $\mathscr{U}$ be an open cover of $X$, and let $M$, $N$ be $(f-d)$ cap-sets for $X$. Then there is a homeomorphism $h$ of $X$ onto itself such that $h(M)=N$ and $h$ is limited by $\mathscr{U}$.

Proof. Using Theorem 2.1 we may, without loss of generality, assume that $X$ is an open subset of $s$. Applying Theorem 1 of [10] there is a star-finite open cover $\left\{U_{i}\right\}_{i=1}^{\infty}$ of $X$ such that for each $i>0, U_{i}$ is a basic open subset of $s$ and there is a $V_{i} \in \mathscr{U}$ such that $d\left(U_{i}, s \mid V_{i}\right)=\varepsilon_{i}>0$. Assume that $\left\{U_{i}\right\}_{i=1}^{\infty}$ is ordered as in Convergence Procedure $B$.

We may regard each $\mathrm{Cl}\left(U_{i}\right)$ (closure taken in $s$ ) as a copy of $s$ together with a finite union of "endslices." Since $M \cap U_{1}$ and $N \cap U_{1}$ are (f-d) cap-sets for $U_{1}$ we can use Lemma 4.3 to get a homeomorphism $h_{1}$ of $X$ onto itself such that $h_{1}\left(M \cap U_{1}\right)=N \cap U_{1}, h_{1}|X| U_{1}=\mathrm{id}$, and $d\left(h_{1}, \mathrm{id}\right)<\varepsilon_{1} / n_{1}$, where $\left\{n_{i}\right\}_{i=1}^{\infty}$ is the sequence of Convergence Procedure B that is associated with $\left\{U_{i}\right\}_{i=1}^{\infty}$.

Proceeding by induction assume that homeomorphisms $\left\{h_{i}\right\}_{i=1}^{j}$ of $X$ onto itself have been defined so that $h_{i}|X| U_{i}=\mathrm{id}$, for $1 \leqq i \leqq j, h_{i+1}\left(h_{i} \circ \ldots \circ h_{1}(M) \cap U_{i}\right)$ $=N \cap U_{i}$, for $1 \leqq i<j$, and $d\left(h_{i}, \mathrm{id}\right)<\left(1 / n_{i}\right) \cdot \min \left(\varepsilon_{1}, \ldots, \varepsilon_{i}\right)$, for $1 \leqq i \leqq j$. Then let $h_{j+1}$ be a homeomorphism of $X$ onto itself such that $h_{j+1}|X| U_{j+1}=\mathrm{id}$, $h_{j+1}\left(h_{j} \circ \ldots \circ h_{1}(M) \cap U_{j+1}\right)=N \cap U_{j+1}$, and $d\left(h_{j+1}, \mathrm{id}\right)<\left(1 / n_{j+1}\right) \cdot \min \left(\varepsilon_{1}, \ldots, \varepsilon_{j+1}\right)$.

We can use Convergence Procedure B to conclude that $h=L \prod_{i=1}^{\infty} h_{i}$ is a homeomorphism of $X$ onto itself such that $h(M)=N$. Moreover we note that if $x \in U_{j} \backslash \bigcup_{i=1}^{j-1} U_{i}$, then $d(h(x), x)<\varepsilon_{j}$. This implies that $h$ is limited by $\mathscr{U}$.

We now prove the corresponding result for $Q$-manifolds. The following lemma takes the place of Theorem 1 of [10] in this case.

Lemma 6.1. Let $X$ be a $Q$-manifold and let $\mathscr{U}$ be an open cover of $X$. Then there is $a$ star-finite open cover $\left\{U_{i}\right\}_{i=1}^{\infty}$ of $X$ which refines $\mathscr{U}$ and for which each $U_{i}$ is homeomorphic to a basic open subset of $I^{\infty}$.

Proof. Since $X$ is a separable, locally compact metric space, it can be written as the union of an increasing sequence $\left\{X_{n}\right\}_{n=1}^{\infty}$ of compacta, each lying in the interior of its successor. 
It is clear that there is a collection $\left\{U_{i}^{1}\right\}_{i=1}^{j_{1}}$ of open subsets of $X$, each homeomorphic to a basic open subset of $I^{\infty}$, such that $\left\{U_{i}^{1}\right\}_{i=1}^{y_{1}}$ covers $X_{1}$, and for each $i$, $U_{i}^{1} \subset X_{2}^{0}$ (the interior of $X_{2}$ ). For each $n>1$ let $\left\{U_{i}^{n}\right\}_{i=1}^{y_{n}}$ be an open cover of $X_{n} \backslash X_{n-1}^{0}$, where each $U_{i}^{n}$ is homeomorphic to a basic open subset of $I^{\infty}$, such that for each $i, \quad U_{i}^{n} \subset X_{n+1}^{0} \backslash X_{n-2}$ (or $U_{i}^{2} \subset X_{3}^{0}$ for $n=2$ ). Then put $\left\{U_{i}\right\}_{i=1}^{\infty}=$ $\left\{U_{i}^{n} \mid 1 \leqq i \leqq j_{n}, n \geqq 1\right\}$ for our required star-finite open cover of $X$.

Since we do not have an open embedding theorem for $Q$-manifolds we will first need a preliminary result for open subsets of $I^{\infty}$. With the aid of Lemma 6.1 a proof of the following result can be given that is almost identical to the proof of Theorem 6.1.

Lemma 6.2. Let $G$ be an open subset of $I^{\infty}$, let $\mathscr{U}$ be an open cover of $G$, and let $M$, $N$ be $(f-d)$ cap-sets for $G$. Then there is a homeomorphism $h$ of $G$ onto itself such that $h(M)=N$ and $h$ is limited by $\mathscr{U}$.

THEOREM 6.2. Let $X$ be a $Q$-manifold, let $\mathscr{U}$ be an open cover of $X$, and let $M, N$ be $(f-d)$ cap-sets for $X$. Then there is a homeomorphism $h$ of $X$ onto itself such that $h(M)=N$ and $h$ is limited by $\mathscr{U}$.

Proof. Using Lemma 6.1 it is clear that there is a star-finite open cover $\left\{U_{i}\right\}_{i=1}^{\infty}$ of $X$ such that for each $i>0, U_{i}$ is homeomorphic to an open subset of $I^{\infty}$ and there is a $V_{i} \in \mathscr{U}$ such that $d\left(U_{i}, X \backslash V_{i}\right)=\varepsilon_{i}>0$.

Using the notation of Theorem 6.1 we need a sequence $\left\{h_{i}\right\}_{i=1}^{\infty}$ of homeomorphisms of $X$ onto itself such that for each $i>0, h_{i}|X| U_{i}=\mathrm{id}, h_{1}\left(M \cap U_{1}\right)=N \cap U_{1}$, $h_{i+1}\left(h_{i} \circ \ldots \circ h_{1}(M) \cap U_{i}\right)=N \cap U_{i}$, and $d\left(h_{i}\right.$, id $)<\left(1 / n_{i}\right) \cdot \min \left(\varepsilon_{1}, \ldots, \varepsilon_{i}\right)$. All of these except the last can be automatically met. The last condition can clearly be met by choosing an appropriate cover of each $U_{i}$, transferring it over to an open cover in $I^{\infty}$, and applying Lemma 6.2. Then $h=L \prod_{i=1}^{\infty} h_{i}$ is our required homeomorphism.

Although it is obvious that any (f-d) cap-set for $s$ is also an (f-d) cap-set for $I^{\infty}$, the corresponding property for $Q$-manifolds is not quite so obvious. This is our next theorem.

THEOREM 6.3. Let $X$ be a $Q$-manifold, let $M \subset X$ be a cap-set for $X$, and let $N$ be an $(f-d)$ cap-set for the F-manifold $X \backslash M$. Then $N$ is an $(f-d)$ cap-set for $X$.

Proof. Using the proof of Lemma 5.6 we know that $X \times B\left(I^{\infty}\right)$ is a cap-set for $X \times I^{\infty}$. Combining the fact that $X$ is homeomorphic to $X \times I^{\infty}$ with Theorem 6.2, there is a homeomorphism $h$ of $X$ onto $X \times I^{\infty}$ such that $h(M)=X \times B\left(I^{\infty}\right)$.

Let $N=\bigcup_{n=1}^{\infty} N_{n}$ be a representation as given in the definition, let $m>0$ be given, let $K$ be a (finite-dimensional) compact subset of $X$, and let $\varepsilon>0$ be given. Using $h$ to transfer the problem to $X \times I^{\infty}$, there is an embedding $f: K \rightarrow X \backslash M$ such that $f \mid K \cap N_{m}=$ id and $d(f$, id) $<\varepsilon / 2$. Using the fact that $N$ is an (f-d) cap-set for $X \backslash M$ there is an integer $n>0$ and an embedding $g: f(K) \rightarrow N_{n}$ such that $g \mid K \cap N_{m}=$ id and $d(g, \mathrm{id})<\varepsilon / 2$. Then $g \circ f$ is our required embedding. 
The next result shows that we can piece together certain portions of (f-d) cap-sets to obtain (f-d) cap-sets.

THEOREM 6.4. Let $X$ be an $F$ or $Q$-manifold and let $U$ be an open subset of $X$. Then the following are true:

(1) If $M$ is an $(f-d)$ cap-set for $U$, then there is an $(f-d)$ cap-set $N$ for $X$ such that $N \cap U=M$.

(2) If $M, N$ are $(f-d)$ cap-sets for $X$, then $(M \backslash U) \cup(N \cap U)$ is an $(f-d)$ cap-set for $X$.

Proof. For the proof of (1) let $N^{\prime}$ be an (f-d) cap-set for $X$. (From Theorem 6.3 we now know that $\mathrm{f}$-d cap-sets exist in $Q$-manifolds.) Using Theorems 6.1 and 6.2 we can obtain a homeomorphism $h$ of $X$ onto itself such that $h\left(N^{\prime} \cap U\right)=M$ and, by a proper choice of a cover of $U, h \mid X \backslash U=\mathrm{id}$. Then $N=h\left(N^{\prime}\right)$ satisfies $N \cap U=M$.

For the proof of (2) let $h$ be a homeomorphism of $X$ onto itself such that $h(M \cap U)=N \cap U$ and $h|X| U=\mathrm{id}$. Then $h(M)=(M \backslash U) \cup(N \cap U)$ is an (f-d) cap-set for $X$.

In the next theorem we prove that ( $\mathrm{f}-\mathrm{d}$ ) cap-sets can be determined locally.

THeOREM 6.5. Let $X$ be an $F$ or $Q$-manifold, let $\left\{U_{i}\right\}_{i=1}^{\infty}$ be an open cover of $X$, and for each $i>0$ let $M_{i}$ be an $(f-d)$ cap-set for $U_{i}$. Then $M=\bigcup_{i=1}^{\infty} M_{i}$ is an $(f-d)$ cap-set for $X$.

Proof. We only sketch the proof for $Q$-manifolds. The proof for $F$-manifolds is similar. Let $\left\{V_{i}\right\}_{i=1}^{\infty}$ be a star-finite open cover of $X$ which is a refinement of $\left\{U_{i}\right\}_{i=1}^{\infty}$ and which is ordered as in Convergence Procedure B. Let $N$ have the (f-d) cap in $X$ and use the techniques of Theorem 6.1 to inductively define a sequence $\left\{h_{i}\right\}_{i=1}^{\infty}$ of homeomorphisms of $X$ onto itself for which $h_{1}\left(M \cap V_{1}\right)=N \cap V_{1}$, $h_{i+1}\left(h_{i} \circ \ldots \circ h_{1}\left(M \cap V_{i+1}\right)\right)=N \cap h_{i} \circ \ldots \circ h_{1}\left(V_{i+1}\right)$, for all $i>0, h_{1}|X| V_{1}=\mathrm{id}$, and $h_{i+1}|X| h_{i} \circ \ldots \circ h_{1}\left(V_{i+1}\right)=\mathrm{id}$, for all $i>0$. We remark that in order to achieve the first and second conditions we have to make use of Lemma 4.1. Then Convergence Procedure D assures us that $h=L \prod_{i=1}^{\infty} h_{i}$ is a homeomorphism of $X$ onto itself which obviously satisfies $h(M)=N$.

We can easily use this result to obtain a generalization of Lemma 4.2 to $F$ and $Q$-manifolds.

THEOREM 6.6. Let $X$ be an $F$ or $Q$-manifold, let $M$ be an $(f-d)$ cap-set for $X$, and let $K$ be a countable union of (finite-dimensional) compact Z-sets in $X$. Then $M \cup K$ is an $(f-d)$ cap-set for $X$.

Proof. In case $X$ is an $F$-manifold we use Theorem 2.1 to get an open embedding $h: X \rightarrow s$. Using Theorem 6.4 there is an (f-d) cap-set $N$ for $s$ such that $N \cap h(X)$ $=h(M)$. By Lemma 4.2 we know that $N \cup h(K)$ is an (f-d) cap-set for $s$, hence $(N \cup h(K)) \cap h(X)=h(M) \cup h(K)$ is an (f-d) cap-set for $h(X)$.

If $X$ is a $Q$-manifold let $U$ be any open subset of $X$ which is homeomorphic to a basic open subset of $I^{\infty}$. Then there is a homeomorphism $f$ of $U$ onto $I^{\infty} \mid W$, where 
$W$ is a finite union of endslices. It is clear that $f(M \cap U)$ is an (f-d) cap-set for $I^{\infty}$ and $f(K \cap U)$ is a countable union of (finite-dimensional) compact $Z$-sets in $I^{\infty}$. From Theorem 4.2 we know that $f(M \cap U) \cup f(K \cap U)$ is an (f-d) cap-set for $I^{\infty}$, hence for $f(U)$. Thus Theorem 6.5 implies that $M \cup K$ is an (f-d) cap-set for $X$.

We can also use Theorem 6.5 to prove that we can remove certain portions of an (f-d) cap-set and still have an (f-d) cap-set.

THEOREM 6.7. Let $X$ be an $F$ or $Q$-manifold, let $M$ be an $(f-d)$ cap-set for $X$, and let $F$ be a $Z$-set in $X$. Then $M \backslash F$ is an $(f-d)$ cap-set for $X$.

Proof. In case $X$ is an $F$-manifold let $U$ be an open subset of $X$ which is homeomorphic to $s$. Then $F \cap U$ is a $Z$-set in $U$. Using Corollary 4.1 we find that $(M \cap U) \backslash(F \cap U)$ has the (f-d) cap in $U$. Then Theorem 6.5 implies that $M \backslash F$ is an (f-d) cap-set for $X$.

If $X$ is a $Q$-manifold let $U$ be an open subset of $X$ for which there is a homeomorphism $h$ of $U$ onto $I^{\infty} \mid W$, where $W$ is a finite union of endslices. We observed in Lemma 5.3 that $f(F \cap U) \cup W$ is a compact $Z$-set in $I^{\infty}$. Using Lemma 4.4 we find that $h(M \cap U) \backslash[h(F \cap U) \cup W]=h(M \cap U) \backslash h(F \cap U)$ is an (f-d) cap-set for $I^{\infty}$, and hence for $h(U)$. Once more Theorem 6.5 implies that $M \backslash F$ is an (f-d) cap-set for $X$.

In our next theorem we show how an (f-d) cap-set of a product relates to the (f-d) cap-sets of the factors.

THEOREM 6.8. Let $X, Y$ both be F-manifolds or both be $Q$-manifolds and let $M \subset X, N \subset Y$ be $(f-d)$ cap-sets. Then $M \times N$ is an $(f-d)$ cap-set for $X \times Y$.

Proof. First assume that $X$ and $Y$ are $F$-manifolds. Let $f_{1}: X \rightarrow s^{\alpha_{1}}$ and $f_{2}: X \rightarrow s^{\alpha_{2}}$ be open embeddings, where $\alpha_{1}$ is the set of odd positive integers and $\alpha_{2}=Z \mid \alpha_{1}$. Then $f_{1}(X) \times f_{2}(Y)$ is an open subset of $s=s^{\alpha_{1}} \times s^{\alpha_{2}}$. We know that there is an (f-d) cap-set $P_{1}$ for $s^{\alpha_{1}}$ such that $P_{1} \cap f_{1}(X)=f_{1}(M)$ and an (f-d) cap-set $P_{2}$ for $s^{\alpha_{2}}$ such that $P_{2} \cap f_{2}(Y)=f_{2}(N)$.

It is obvious that there exist canonical (f-d) cap-sets $R_{1}$ for $s^{\alpha_{1}}$ and $R_{2}$ for $s^{\alpha_{2}}$ such that $R_{1} \times R_{2}$ is an (f-d) cap-set for $s$. This implies that $P_{1} \times P_{2}$ is an (f-d) cap-set for $s$ and thus $\left(P_{1} \times P_{2}\right) \cap\left(f_{1}(X) \times f_{2}(Y)\right)=f_{1}(M) \times f_{2}(N)$ is an (f-d) cap-set for $f_{1}(X) \times f_{2}(Y)$.

Now assume that $X$ and $Y$ are $Q$-manifolds. Using Lemmas 5.3 and 5.5 we find that if $K_{1} \subset X$ is a compact $Z$-set in $X$ and $K_{2} \subset Y$ is a compact $Z$-set in $Y$, then $K_{1} \times K_{2}$ is a $Z$-set in $X \times Y$. If we use the techniques involved in the proof of Lemma 5.6 it follows that if $M$ and $N$ are cap-sets, then $M \times N$ is a cap-set for $X \times Y$. Thus all we have left to do is the case in which $M$ and $N$ are f-d cap-sets.

Let $M^{\prime}$ be a cap-set for $X$ and let $N^{\prime}$ be a cap-set for $Y$. It clearly follows from the techniques involved in the proof of Lemma 5.6 that $X \times N^{\prime}$ and $M^{\prime} \times Y$ are cap-sets for $X \times Y$. Using Theorem 6.6 we find that $\left(X \times N^{\prime}\right) \cup\left(M^{\prime} \times Y\right)$ is a capset for $X \times Y$. 
Now choose f-d cap-sets $M^{\prime \prime}$ for $X \backslash M^{\prime}$ and $N^{\prime \prime}$ for $Y \backslash N^{\prime}$. Using what we proved above we know that $M^{\prime \prime} \times N^{\prime \prime}$ is an f-d cap-set for $\left(X \backslash M^{\prime}\right) \times\left(Y \backslash N^{\prime}\right)$. Since $(X \times Y) \backslash\left[\left(X \times N^{\prime}\right) \cup\left(M^{\prime} \times Y\right)\right]=\left(X \backslash M^{\prime}\right) \times\left(Y \backslash N^{\prime}\right)$ it follows from Theorem 6.3 that $M^{\prime \prime} \times N^{\prime \prime}$ is an $\mathrm{f}-\mathrm{d}$ cap-set for $X \times Y$. Then we can clearly use Theorem 6.2 to relate this to $M$ and $N$.

We can use Theorem 6.7 to characterize $Z$-sets in (f-d) cap-sets.

THEOREM 6.9. Let $X$ be an F-manifold and let $M$ be an $(f-d)$ cap-set for $X$. A necessary and sufficient condition that a closed subset $K$ of $M$ has Property $\mathrm{Z}$ in $M$ is that $K$ be strongly negligible in $M$.

Proof. If $K$ is strongly negligible in $M$, then we can use the proof of Theorem 2.20 to prove that $K$ is a $Z$-set in $M$.

Thus assume that $K$ is a $Z$-set in $M$ and let $\left\{U_{i}\right\}_{i=1}^{\infty}$ be an open cover of $M$. For each $i>0$ let $V_{i}$ be an open subset of $X$ satisfying $V_{i} \cap M=U_{i}$. Without loss of generality we may assume that $X=\bigcup_{i=1}^{\infty} V_{i}$.

By Lemma $5.10, \bar{K}$ (closure in $X$ ) is a $Z$-set in $X$. Using Theorem 6.7 it follows that $M \backslash \bar{K}$ is an (f-d) cap-set for $X$. By Theorem 6.1 there exists a homeomorphism $h$ of $X$ onto itself such that $h(M)=M \backslash \bar{K}$ and $h$ is limited by $\left\{V_{i}\right\}_{i=1}^{\infty}$. Thus $K$ is strongly negligible in $M$.

7. Infinite-deficiency in manifolds. In [13] a proof of the following theorem is given.

THEOREM 7.1. Let $X$ be an F-manifold and let $F$ be a closed subset of $X$. A necessary and sufficient condition that $F$ have Property $\mathrm{Z}$ in $X$ is that there exists a homeomorphism $h$ of $X$ onto $X \times s$ such that $\pi_{s}(h(F))$ has infinite deficiency.

The technique involved in the proof of Theorem 7.1 is to use Theorem 2.10 and then to modify the copy of $F$ in $X \times s$ so that its projection in $s$ has infinite deficiency.

In our next theorem we give a similar characterization of Property $\mathrm{Z}$ in $Q$ manifolds. As a corollary we get an easy proof of Theorem 7.1.

TheOrem 7.2. Let $X$ be a $Q$-manifold and let $F$ be a closed subset of $X \times I^{\infty}$.A necessary and sufficient condition that $F$ have Property $\mathrm{Z}$ in $X \times I^{\infty}$ is that there exists a homeomorphism $h$ of $X \times I^{\infty}$ onto itself taking $F$ onto a set whose projection in $I^{\infty}$ has infinite deficiency. Moreover, we can construct $h$ so that $h\left(X \times B\left(I^{\infty}\right)\right)$ $=X \times B\left(I^{\infty}\right)$.

Proof. The step from infinite deficiency to Property $\mathrm{Z}$ is easy and is similar to the proof of Theorem 9.1 of [3]. Thus assume that $F$ is a $Z$-set in $X \times I^{\infty}$. Since $X \times B\left(I^{\infty}\right)$ is a cap-set for $X \times I^{\infty}$, it follows from Theorem 6.7 that $\left(X \times B\left(I^{\infty}\right)\right) \backslash F$ is also a cap-set for $X \times I^{\infty}$. Using Theorem 6.2 there is a homeomorphism $f_{1}$ of $X \times I^{\infty}$ onto itself such that $f_{1}\left(\left(X \times B\left(I^{\infty}\right)\right) \backslash F\right)=X \times B\left(I^{\infty}\right)$. This gives us

$$
f_{1}(F) \cap\left(X \times B\left(I^{\infty}\right)\right)=\varnothing .
$$


Let $j>0$ be an integer and for each $x \in X \times \prod_{i \neq j} I_{i}$ let $U_{x} \times\left(\delta_{x}, 1\right]$ be an open subset of $\left(X \times \prod_{i \neq j} I_{i}\right) \times I_{j}$ containing $(x, 1)$ such that $\left(U_{x} \times\left(\delta_{x}, 1\right]\right) \cap f_{1}(F)=\varnothing$. Note that $\left\{U_{x} \mid x \in X \times \prod_{i \neq j} I_{i}\right\}$ is an open cover of $X \times \prod_{i \neq j} I_{i}$. Thus we can use Lemma 6.1 to get a star-finite open refinement $\left\{U_{i}\right\}_{i=1}^{\infty}$ of $\left\{U_{x} \mid x \in X \times \prod_{i \neq j} I_{i}\right\}$ which covers $X \times \prod_{i \neq j} I_{i}$. Moreover we may assume that the $U_{i}$ 's are indexed as in Convergence Procedure B. It is clear that for each $i>0$ there is a number $\delta_{i}>0$ such that $\frac{1}{2} \leqq \delta_{i}<1$ and $\left(U_{i} \times\left(\delta_{i}, 1\right]\right) \cap f_{1}(F)=\varnothing$.

Now construct a closed cover $\left\{C_{i}\right\}_{i=1}^{\infty}$ of $X \times \prod_{i \neq j} I_{i}$ such that $C_{i} \subset U_{i}$, for all $i>0$. For each $i>0$ let $\varphi_{i}: X \times \prod_{i \neq j} I_{i} \rightarrow[0,1]$ be a continuous function such that $\varphi_{i}(x)=1$ for $x \in C_{i}$ and $\varphi_{i}(x)=0$ for $x \in X \times \prod_{i \neq j} I_{i} \mid U_{i}$. For each $i>0$ we now construct a homeomorphism $g_{i}$ of $\left(X \times \prod_{i \neq j} I_{i}\right) \times I_{j}$ onto itself which slides points linearly in the $I_{j}$-direction as follows: if $x \in X \times \prod_{i \neq j} I_{i}$, then $\{x\} \times I_{j}$ is taken linearly onto itself such that $g_{i}\left(x, \delta_{i}\right)=\left(x, \delta_{i}\left(1-\varphi_{i}(x)\right)+\left(\frac{1}{2}\right) \varphi_{i}(x)\right)$. Applying Convergence Procedure $\mathrm{C}$ to $\left\{g_{i}\right\}_{i=1}^{\infty}$ let $g=L \prod_{i=1}^{\infty} g_{i}$, which is a homeomorphism of $X \times I^{\infty}$ onto itself satisfying $g \circ f_{1}(F) \subset\left(X \times \prod_{i \neq j} I_{i}\right) \times\left[-1, \frac{1}{2}\right]$. Using similar techniques we can construct a homeomorphism $g^{\prime}$ of $X \times I^{\infty}$ onto itself which slides points only in the $I_{j}$-direction such that $g^{\prime} \circ g \circ f_{1}(F) \subset X \times \prod_{i \neq j} I_{i} \times\left[-\frac{1}{2}, \frac{1}{2}\right]$. Then put $g^{j}=g^{\prime} \circ g$. We note that $g^{j}$ is a homeomorphism of $X \times I^{\infty}$ onto itself which satisfies $g^{j} \circ f_{1}(F) \subset X \times \prod_{i \neq j} I_{i} \times\left[-\frac{1}{2}, \frac{1}{2}\right], \pi_{X} \circ g^{j}(x, t)=x$, and

$$
\tau_{i} \circ \pi_{I}^{\infty} \circ g^{j}(x, t)=\tau_{i}(t),
$$

for all $i \neq j$ and $(x, t) \in X \times I^{\infty}$.

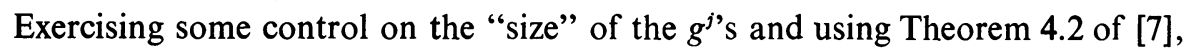
it is now clear that $f_{2}=L \prod_{j=1}^{\infty} g^{j}$ gives a homeomorphism $f_{2}$ of $X \times I^{\infty}$ onto itself such that $f_{2}\left(f_{1}(F)\right) \subset X \times \prod_{i=1}^{\infty}\left[-\frac{1}{2}, \frac{1}{2}\right]$ and $f_{2}\left(X \times B\left(I^{\infty}\right)\right)=X \times B\left(I^{\infty}\right)$. Using Theorem 3.5 of [2] there is a $\beta^{*}$-homeomorphism $\psi: I^{\infty} \rightarrow I^{\infty}$ such that $\psi\left(\prod_{i=1}^{\infty}\left[-\frac{1}{2}, \frac{1}{2}\right]\right)$ has infinite deficiency. Then $f_{3}=\mathrm{id} \times \psi$ gives a homeomorphism of $X \times I^{\infty}$ onto itself such that $\pi_{I} \circ f_{3}\left(X \times \prod_{i=1}^{\infty}\left[-\frac{1}{2}, \frac{1}{2}\right]\right)$ has infinite deficiency and $f_{3}\left(X \times B\left(I^{\infty}\right)\right)=X \times B\left(I^{\infty}\right)$.

Now $f_{3} \circ f_{2} \circ f_{1}$ is a homeomorphism of $X \times I^{\infty}$ onto itself such that $\pi_{I} \circ \circ f_{3} \circ f_{2} \circ f_{1}(F)$ has infinite deficiency. We also note that

$$
f_{3} \circ f_{2} \circ f_{1}\left[F \cap\left(X \times B\left(I^{\infty}\right)\right)\right] \subset X \times s
$$

and is a countable union of compact sets. Using Corollary 5.7 of [2] for pushing a $\sigma$-compact subset of $s$ to $B\left(I^{\infty}\right)$ there is a homeomorphism $f_{4}$ of $X \times I^{\infty}$ onto itself such that $\pi_{I} \circ \circ f_{4} \circ f_{3} \circ f_{2} \circ f_{1}(F)$ has infinite deficiency and $f_{4} \circ f_{3} \circ f_{2} \circ f_{1}\left(X \times B\left(I^{\infty}\right)\right)$ $=X \times B\left(I^{\infty}\right)$. Then $h=f_{4} \circ f_{3} \circ f_{2} \circ f_{1}$ satisfies our requirements.

COROLlaRY 7.1. If $X$ is an F-manifold and $F$ is a Z-set in $X \times s$, then there is a homeomorphism $h$ of $X \times s$ onto itself such that $\pi_{s} \circ h(F)$ has infinite deficiency.

Proof. Using Theorem 5.1 there is a $Q$-manifold $Y$ containing $X \times s$ such that $Y \backslash(X \times s)$ is a cap-set for $Y$. This means that there is a homeomorphism $f$ of $Y$ onto 
$Y \times I^{\infty}$ such that $f(X \times s)=Y \times s$. Then $f(F)$ is a $Z$-set in $Y \times s$. By Lemma 5.7 we know that $\mathrm{Cl}(f(F))$ (closure taken in $Y \times I^{\infty}$ ) is a Z-set in $Y \times I^{\infty}$. Thus there is a homeomorphism $g$ of $Y \times I^{\infty}$ onto itself such that $g(Y \times s)=Y \times s$ and $g(\mathrm{Cl}(f(F)))$ has infinite deficiency. We can write $s=s_{1} \times s_{2}$, where $s_{1}, s_{2}$ are copies of $s$ and $g(\mathrm{Cl}(f(F)))$ is infinitely deficient in the $s_{2}$ factor. Then the following diagram, with appropriate restrictions on the homeomorphisms involved, gives our desired homeomorphism.

$$
X \times s \stackrel{f}{\longrightarrow} Y \times s \stackrel{g}{\longrightarrow} Y \times s=\left(Y \times s_{1}\right) \times s_{2} \stackrel{f^{-1} x \text { id }}{\longrightarrow} X \times s .
$$

8. Product and factor theorems. The first result we establish is a relationship between cap-sets and $\mathrm{f}$-d cap-sets in $F$-manifolds. For its proof we will require two preliminary lemmas.

The first lemma gives sufficient conditions in order that a subset of $I^{\infty}$ be a capset for $I^{\infty}$. These conditions are also necessary, and in [5] Anderson used them for a definition of cap-set.

LEMMA 8.1. Let $M \subset I^{\infty}$ be a countable union of Z-sets in $I^{\infty}$ which satisfies the property that for each $\varepsilon>0$ and each pair $A, B$ of compact subsets of $I^{\infty}$, with $A \subset B \cap M$, there is an embedding $h: B \rightarrow M$ such that $h \mid A=\mathrm{id}$ and $d(h, \mathrm{id})<\varepsilon$. Then $M$ is a cap-set for $I^{\infty}$.

Proof. Let $N$ be a cap-set for $I^{\infty}$ and write $N=\bigcup_{n=1}^{\infty} N_{n}$, as in the definition. Then there is an embedding $f_{1}: N_{1} \rightarrow M$. Since $f_{1}\left(N_{1}\right)$ obviously has Property $\mathrm{Z}$ in $I^{\infty}$ we can use Lemma 2.1 of [13] to extend $f_{1}$ to a homeomorphism $g_{1}$ of $I^{\infty}$ onto itself.

Using the above properties of $M$ we can inductively obtain a sequence $\left\{g_{i}\right\}_{i=1}^{\infty}$ of homeomorphisms of $I^{\infty}$ onto itself such that

(1) for each $i>0, g_{i} \circ \ldots \circ g_{1}\left(N_{i}\right) \subset M$,

(2) for each $i>0, g_{i+1} \mid g_{i} \circ \ldots \circ g_{1}\left(N_{i}\right)=\mathrm{id}$, and

(3) for each $i>1, d\left(g_{i}\right.$, id $)<\min \left(\left(3^{-i}\right),\left(3^{-i}\right) \cdot \eta\left(g_{i-1} \circ \cdots \circ g_{1}, 2^{-i}\right)\right)$.

Applying Convergence Procedure A we find that $g=L \prod_{i=1}^{\infty} g_{i}$ gives a homeomorphism of $I^{\infty}$ onto itself such that $g(N) \subset M$. Since $g(N)$ is a cap-set for $I^{\infty}$ it follows from Lemma 4.2 that $M$ is also.

LEMMA 8.2. For each $n>0$ define

$$
M_{n}=\left\{x \in I^{\infty} \mid \tau_{i}(x)=0, \text { for } i \geqq 2 n-1 \text { and odd }\right\} .
$$

Then $M=\bigcup_{n=1}^{\infty} M_{n}$ has the cap in $I^{\infty}$.

Proof. We will prove that $M$ has the properties of Lemma 8.1. Thus let $A$ be a compact subset of $M$ and let $\varepsilon>0$ be given. It will clearly be sufficient to prove the existence of an embedding $h: I^{\infty} \rightarrow M$ satisfying $h \mid A=$ id and $d(h$, id $)<\varepsilon$.

Let $\mathscr{U}$ be an open cover of $I^{\infty} \mid A$ such that for elements $U \in \mathscr{U}$, diam $(U)$ "becomes small" as $d(U, A)$ "becomes small." Then let $\left\{U_{i}\right\}_{i=1}^{\infty}$ be a star-finite 
refinement of $\mathscr{U}$ such that each $U_{i}$ is a basic open set in $I^{\infty}$ and assume that $\left\{U_{i}\right\}_{i=1}^{\infty}$ is ordered as in Convergence Procedure B. Choose an odd integer $m$ such that $\left(\sum_{i=m}^{\infty} 2^{2-i}\right)^{1 / 2}<\varepsilon$. We will construct $h$ so that $h$ does not affect the first $m-1$ coordinates of any point.

Using the simplified geometry of the basic open sets $U_{i}$, we can rotate coordinates in an obvious manner to obtain embeddings $h_{i}: I^{\infty} \rightarrow I^{\infty}$ such that for each $i>0, h_{i}\left(U_{i}\right) \subset M, h_{i}\left|I^{\infty}\right| U_{i}=\mathrm{id}$, and $h_{i}$ does not affect the first $m-1$ coordinates of any point. Each point $x$ of $I^{\infty}$ is moved only a finite number of times in the sequence $h_{1}(x), h_{2} \circ h_{1}(x), h_{3} \circ h_{2} \circ h_{1}(x), \ldots$ Thus $h(x)=\lim _{i \rightarrow \infty} h_{i} \circ \ldots \circ h_{1}(x)$ defines an embedding $I^{\infty}$ into $M$ such that $h \mid A=\mathrm{id}$ and $d(h, \mathrm{id})<\varepsilon$.

THEOREM 8.1. Let $X$ be an F-manifold and let $M \subset X$ be an $f$-d cap-set for $X$. Then $M \times I^{\infty}$ is a cap-set for $X \times I^{\infty}$.

Proof. We will use Theorem 6.5 by showing that if $U$ is an open subset of $X$ which is homeomorphic to $s$, then $(M \cap U) \times I^{\infty}$ has the cap in $U \times I^{\infty}$.

We know that there is a homeomorphism $f$ of $U$ onto $s$ satisfying $f(M \cap s)=\sigma$. Thus it will suffice to prove that $\sigma \times I^{\infty}$ has the cap in $s \times I^{\infty}$. Let $I_{1}^{\infty}=\prod\left\{I_{i} \mid i\right.$ odd $\}$ and $I_{2}^{\infty}=\prod\left\{I_{i} \mid i\right.$ even $\}$. Let $A$ be the set of all elements of $I_{1}^{\infty}$ having at most finitely many nonzero coordinates. Using Lemma 4.2 we know that $A$ has the $\mathrm{f}-\mathrm{d}$ cap in $I_{1}^{\infty}$. Lemma 8.2 shows that $A \times I_{2}^{\infty}$ has the cap on $I_{1}^{\infty} \times I_{2}^{\infty}$. There is a homeomorphism $g$ of $I_{1}^{\infty}$ onto itself such that $g(A)=\sigma_{1}$, where $\sigma_{1}$ is the set of all points in $s_{1}=\prod\left\{I_{i}^{0} \mid i\right.$ odd $\}$ having at most finitely many nonzero coordinates. Thus $\sigma_{1} \times I_{2}^{\infty}$ has the cap in $I_{1}^{\infty} \times I_{2}^{\infty}$ and hence in $s_{1} \times I_{2}^{\infty}$. This is what we wished to prove.

Corollary 8.1. Let $X$ be an F-manifold, let $M \subset X$ be an $f$-d cap-set for $X$, and let $N \subset X$ be a cap-set for $X$. Then $M \times I^{\infty}$ is homeomorphic to $N$.

Proof. This follows immediately from Theorems 6.1 and 8.1 .

We know from Theorem 2.8 that given any $F$-manifold $X$, there is a countable locally-finite simplicial complex $K$ such that $X$ is homeomorphic to $|K| \times s$. We use this in our next theorem.

THEOREM 8.2. Let $X$ be an F-manifold and let $K$ be a complex as above. If $M \subset X$ is a cap-set (or $f$-d cap-set) for $X$, then $M$ is homeomorphic to $|K| \times \Sigma$ (or $|K| \times \sigma)$.

Proof. All that we need to do is prove that $|K| \times \sigma$ has the $\mathrm{f}$-d cap in $|K| \times s$, since the proof that $|K| \times \Sigma$ has the cap in $|K| \times s$ is easy and resembles the proof of Lemma 5.6.

Write $|K|=\bigcup_{n=1}^{\infty}\left|K_{n}\right|$, where $\left|K_{n}\right|$ is a finite complex contained in the interior of $\left|K_{n+1}\right|$, and write $\sigma=\bigcup_{n=1}^{\infty} M_{n}$ so as to satisfy the definition of an f-d cap-set for $s$. We will show that the representation $|K| \times \sigma=\bigcup_{n=1}^{\infty}\left(\left|K_{n}\right| \times M_{n}\right)$ satisfies the definition for $|K| \times \sigma$ to have the $\mathrm{f}-\mathrm{d}$ cap in $|K| \times s$. To this end let $m>0$ be an integer, let $F$ be a finite-dimensional compact subset of $|K| \times s$, and let $\varepsilon>0$ be given.

If $C$ is any compact subset of $s$, then there is a homeomorphism of $s$ onto 
$s \times[0,1)$ taking $C$ into $s \times\{0\}$. This means that there is an isotopy $G: s \times I \rightarrow s$ such that $G_{0}=\mathrm{id}, d\left(x, G_{t}(x)\right)<\varepsilon / 3$, for all $t \in I$ and $x \in s$, and $G_{t}(s) \cap M_{m}=\varnothing$, for all $t>0$. For each $x \in F$ define $f(x)=G_{t} \circ \pi_{s}(x)$, where

$$
t=d\left(x,\left|K_{m}\right| \times M_{m}\right) /\left(1+d\left(x,\left|K_{m}\right| \times M_{m}\right)\right) .
$$

Then $f: F \rightarrow s$ is a continuous function such that $d\left(f, \pi_{s}\right)<\varepsilon / 3$ and $f\left(F \mid\left(\left|K_{m}\right| \times M_{m}\right)\right)$ $\cap M_{m}=\varnothing$.

In [8] it is shown that if $X$ is any $F$-manifold, $A$ is a topologically complete separable metric space, $\mathscr{U}$ is an open cover of $X$, and $g: A \rightarrow X$ is a continuous function, then there is an embedding $\tilde{g}: A \rightarrow X$ such that $\tilde{g}$ is $\mathscr{U}$-close to $g$ (i.e. for each $a \in A$ there exists an element of $\mathscr{U}$ containing both $\tilde{g}(a)$ and $g(a)$ ). If we let $X=s \backslash M_{m}$ and choose an appropriate cover of $X$, then we can use this result to obtain a continuous function $\tilde{f}: F \rightarrow s$ such that $\tilde{f}|| K_{m}\left|\times M_{m}=\pi_{s}\right|\left|K_{m}\right| \times M_{m}$, $\tilde{f}|F|\left(\left|K_{m}\right| \times M_{m}\right)$ is an embedding, and $d(\tilde{f}, f)<\varepsilon / 3$.

Note that $\tilde{f}(F)$ is a finite-dimensional compact subset of $s$. Thus there exists an integer $n>0$ and an embedding $g: f(F) \rightarrow M_{n}$ such that $g \mid f(F) \cap M_{m}=$ id and $d(g$, id $)<\varepsilon / 3$. Now let $n^{\prime} \geqq n$ be an integer such that $\pi_{|K|}(F) \subset\left|K_{n^{\prime}}\right|$. Define $h$ : $F \rightarrow\left|K_{n^{\prime}}\right| \times M_{n^{\prime}}$ by $h(x)=\left(\pi_{|K|}(x), g \circ \tilde{f}(x)\right)$, for all $x \in F$. Then $h$ is an embedding, $h \mid F \cap\left(\left|K_{m}\right| \times M_{m}\right)=\mathrm{id}$, and $d(h, \mathrm{id})<\varepsilon$, as we wanted.

CoRollary 8.2. Let $X$ be an $F$ or $Q$-manifold, let $M \subset X$ have the $f$-d cap, and let $N \subset X$ have the cap. Then $X, M$, and $N$ are all of the same homotopy type.

Proof. For $X$ an $F$-manifold the proof follows from Theorem 8.2. If $X$ is a $Q$-manifold we have already shown (Lemma 5.6 and Theorem 6.2) that $N$ is homeomorphic to $X \times \Sigma$, and hence has the same homotopy type as $X$. Using Corollary 5.1 we find that $M$ has the same homotopy type as $N$.

Corollary 8.3. Let $X$ be a $Q$-manifold and let $M \subset X$ have the $(f-d)$ cap. Then $X \backslash M$ is an F-manifold of the same homotopy type as $X$.

Proof. If $N$ is an (f-d) cap-set for $X \backslash M$, then Corollary 8.2 implies that $N$ and $X \backslash M$ have the same homotopy type. From Theorem 6.2 it follows that $N$ is homeomorphic to $M$, and from Corollary 8.2 it follows that $M$ and $X$ have the same homotopy type.

From Theorem 2.5 we know that if $K$ is any countable locally-finite simplicial complex, then $|K| \times s$ is an $F$-manifold. We use this to prove a corresponding property for $\sigma$ and $\Sigma$-manifolds.

COROLlaRY 8.4. If $K$ is any countable locally-finite simplicial complex, then $|K| \times \sigma$ is a $\sigma$-manifold and $|K| \times \Sigma$ is a $\Sigma$-manifold.

Proof. From Theorem 8.2 we know that $|K| \times \sigma$ is an f-d cap-set for $|K| \times s$ and $|K| \times \Sigma$ is a cap-set for $|K| \times s$. Then Lemma 5.4 implies that $|K| \times \sigma$ is a $\sigma$-manifold and $|K| \times \Sigma$ is a $\Sigma$-manifold. 
We can easily establish some factor theorems for (f-d) cap-sets in $F$-manifolds.

THEOREM 8.3. Let $X$ be an F-manifold, let $M$ be an $f$-d cap-set for $X$, and let $I^{n}$ be any $n$-cell. Then $M, M \times \sigma$, and $M \times I^{n}$ are all homeomorphic.

Proof. Using Theorem 6.8 it follows that $M \times \sigma$ is an $\mathrm{f}-\mathrm{d}$ cap-set for $X \times s$. This implies that $M$ and $M \times \sigma$ are homeomorphic.

Since $X$ and $X \times I^{\infty}$ are homeomorphic it follows that $X$ and $X \times I^{n}$ are also homeomorphic. Thus to show that $M$ and $M \times I^{n}$ are homeomorphic all we have to do is prove that $M \times I^{n}$ is an f-d cap-set for $X \times I^{n}$. Using Theorem 6.5 it is clearly sufficient to prove that if $U$ is any open subset of $X$ which is homeomorphic to $s$, then $(M \cap U) \times I^{n}$ is an $\mathrm{f}-\mathrm{d}$ cap-set for $U \times I^{n}$. Thus the problem reduces to the following: Show that $\sigma \times I^{n}$ is an f-d cap-set for $s \times I^{n}$.

Let $s_{1}=\prod\left\{I_{i}^{0} \mid i>n\right\}$ and $\sigma_{1}=\left\{x \in s_{1} \mid x\right.$ has at most finitely many nonzero coordinates\}. Then Lemma 4.2 implies $\sigma_{1} \times I^{n}$ is an f-d cap-set for $I^{\infty}=I_{1}^{\infty} \times I^{n}$, where $I_{1}^{\infty}=\prod\left\{I_{i} \mid i>n\right\}$. Thus $\sigma_{1} \times I^{n}$ is an f-d cap-set for $s_{1} \times I^{n}$, which is what we wished to prove.

Corollary 8.5. Let $X$ be an F-manifold and let $M$ be a cap-set for $X$. Then $M, M \times \Sigma$, and $M \times I^{\infty}$ are all homeomorphic.

Proof. It follows from Corollary 8.1 that $M$ is homeomorphic to $N \times I^{\infty}$, where $N$ is an f-d cap-set for $X$. Thus $M$ and $M \times I^{\infty}$ are homeomorphic. It then follows that $\Sigma$ and $\sigma \times I^{\infty}$ are homeomorphic. Thus (with " $\simeq$ " meaning "is homeomorphic to")

$$
M \times \Sigma \simeq\left(N \times I^{\infty}\right) \times\left(\sigma \times I^{\infty}\right) \simeq(N \times \sigma) \times I^{\infty} \simeq N \times I^{\infty} \simeq M .
$$

9. Permuting sets which have the (f-d) cap. The following result is a generalization of Lemma 4.3. Since its proof is similar, except that we are handling several subsets of $I^{\infty}$ having the (f-d) cap at one time, we omit its proof.

LEMMA 9.1. Let $\left\{M_{i}\right\}_{i=1}^{n}$ ( $n$ finite or $\infty$ ) be a collection of disjoint subsets of $I^{\infty}$ which have the $(f-d)$ cap in $I^{\infty}$ and let $\left\{N_{i}\right\}_{i=1}^{n}$ be a collection of disjoint subsets of $I^{\infty}$ which have the $(f-d)$ cap in $I^{\infty}$. If $K \subset B\left(I^{\infty}\right) \backslash\left[\bigcup_{i=1}^{n}\left(M_{i} \cup N_{i}\right)\right]$ is compact, then there is a homeomorphism $h$ of $I^{\infty}$ onto itself such that $h \mid K=\mathrm{id}$ and $h\left(M_{i}\right)=N_{i}$, for all $i$. If $\bigcup_{i=1}^{n}\left(M_{i} \cup N_{i}\right) \subset s$, then we may additionally require that $h$ be a $\beta^{*}$-homeomorphism.

We can easily apply the techniques of $\S 6$ to prove the following theorem.

THEOREM 9.1. Let $\left\{M_{i}\right\}_{i=1}^{n}$ ( $n$ finite or $\infty$ ) be a collection of disjoint subsets of an $F$ or Q-manifold $X$ which have the $(f-d)$ cap in $X$ and let $\left\{N_{i}\right\}_{i=1}^{n}$ be a collection of disjoint subsets of $X$ which have the $(f-d)$ cap in $X$. Then there is a homeomorphism $h$ of $X$ onto itself such that $h\left(M_{i}\right)=N_{i}$, for all $i$.

In our next theorem we prove that any finite collection of $n$ disjoint (f-d) cap-sets for an $F$ or $Q$-manifold can be permuted with a period $n$ homeomorphism. 
THEOREM 9.2. Let $\left\{M_{i}\right\}_{i=1}^{n}$ be a finite collection of disjoint subsets of an $F$ or $Q-$ manifold $X$, with each $M_{i}$ having the $(f-d)$ cap in $X$. Then there is a period $n$ homeomorphism $h$ of $X$ onto itself such that $h\left(M_{i}\right)=M_{i+1}$, for $1 \leqq i \leqq n-1$, and $h\left(M_{n}\right)=M_{1}$.

Proof. We only treat the case in which $X$ is an $F$-manifold and each $M_{i}$ is a capset for $X$. The other cases are similar.

Let $\left\{\left[a_{i}, b_{i}\right] \times\left[c_{i}, d_{i}\right]\right\}_{i=1}^{n}$ be a collection of disjoint rectangles in $(-1,1) \times(-1,1)$ and let $f$ be a homeomorphism of $(-1,1) \times(-1,1)$ onto itself of period $n$ such that $f\left(\left[a_{i}, b_{i}\right] \times\left[c_{i}, d_{i}\right]\right)=\left[a_{i+1}, b_{i+1}\right] \times\left[c_{i+1}, d_{i+1}\right]$, for $1 \leqq i \leqq n-1$, and

$$
f\left(\left[a_{n}, b_{n}\right] \times\left[c_{n}, d_{n}\right]\right)=\left[a_{1}, b_{1}\right] \times\left[c_{1}, d_{1}\right] .
$$

For $1 \leqq i \leqq n$ let $C_{i}$ be the subset of $s$ which consists of all points $x \in s$ such that $\tau_{2 j-1}(x) \in\left[a_{i}, b_{i}\right]$ and $\tau_{2 j}(x) \in\left[c_{i}, d_{i}\right]$, for all $j>0$.

For $1 \leqq i \leqq n$ let $N_{i}$ be the basic core set in $s$ structured over $C_{i}$. Then $\left\{N_{i}\right\}_{i=1}^{n}$ is a collection of disjoint cap-sets for $s$. Let $g$ be the homeomorphism of $s$ onto itself such that $\tau_{\{2 j-1,2 j\}} \circ g(x)=f\left(\tau_{\{2 j-1,2 j\}}(x)\right)$, for all $j>0$. Then $g$ is a homeomorphism of $s$ onto itself of period $n$ such that $g\left(N_{i}\right)=N_{i+1}$, for $1 \leqq i \leqq n-1$, and $g\left(N_{n}\right)=N_{1}$.

Now let $M$ be a cap-set for $X$ and use Theorem 6.8 to conclude that $\left\{M \times N_{i}\right\}_{i=1}^{n}$ is a collection of disjoint cap-sets for $X \times s$. Also id $\times g: X \times s \rightarrow X \times s$ gives a homeomorphism of period $n$ which permutes the collection $\left\{M \times N_{i}\right\}_{i=1}^{n}$. Since $X$ is homeomorphic to $X \times s$ this means that there is a collection $\left\{N_{i}^{\prime}\right\}_{i=1}^{n}$ of disjoint cap-sets for $X$ and a period $n$ homeomorphism $h^{\prime}$ of $X$ onto itself which permutes $\left\{N_{i}^{\prime}\right\}_{i=1}^{n}$.

Using Theorem 9.1 there is a homeomorphism $\varphi$ of $X$ onto itself such that $\varphi\left(M_{i}\right)=N_{i}^{\prime}$, for $1 \leqq i \leqq n$. Then $h=\varphi^{-1} \circ h^{\prime} \circ \varphi$ satisfies our required properties.

Using similar devices we can also permute a countably infinite collection of disjoint (f-d) cap-sets for an $F$ or $Q$-manifold.

THEOREM 9.3. Let $\left\{M_{i}\right\}_{i=-\infty}^{\infty}$ be a collection of disjoint subsets of an $F$ or $Q-$ manifold $X$, each $M_{i}$ having the $(f-d)$ cap in $X$. Then there is a homeomorphism $h$ of $X$ onto itself such that $h\left(M_{i}\right)=M_{i+1}$, for all $i$.

Proof. Once more we only treat the case in which $X$ is an $F$-manifold and each $M_{i}$ is a cap-set for $X$.

Let $\left\{\left[a_{i}, b_{i}\right]\right\}_{i=1}^{\infty}$ be a collection of disjoint subintervals of $(-1,1)$ such that $b_{i}<a_{i+1}$, for all $i$, and let $f$ be a homeomorphism of $(-1,1)$ onto itself such that $f\left(\left[a_{i}, b_{i}\right]\right)=\left[a_{i+1}, b_{i+1}\right]$, for all $i$. For each $i$ let $C_{i}$ be the subset of $s$ consisting of all points $x \in s$ for which $\tau_{j}(x) \in\left[a_{l}, b_{i}\right]$, for all $j>0$. As in Theorem 9.2 let $N_{i}$ be the basic core set in $s$ structured over $C_{i}$. It is now clear that we can use techniques like those used in the proof of Theorem 9.2 to complete the proof of this theorem.

10. Open embedding theorems for $\sigma$ and $\Sigma$-manifolds. The main result of this section is Theorem 10.3 in which we prove that any $\sigma$ (or $\Sigma$ )-manifold can be embedded as an open subset of $\sigma$ (or $\Sigma$ ). Our proof makes use of Henderson's open 
embedding theorem for $F$-manifolds (Theorem 2.3). Our strategy is to first embed the $\sigma$ (or $\Sigma$ )-manifold as an f-d cap (or cap)-set for an $F$-manifold (Theorem 10.2), and then apply Henderson's result.

In this and the next section we will need the following well-known result which is due to Lavrent'ev (see [16, p. 235]).

THEOREM 10.1. Each homeomorphism between subspaces $A$ and $B$ of topologically complete metric spaces $X$ and $Y$, respectively, can be extended to a homeomorphism between $G_{\delta}$-subsets of $X$ and $Y$.

The following result gives a technique for finding $F$-manifolds in topologically complete metric spaces. We will also use this in the next section.

LEMMA 10.1. Let $X$ be an F-manifold, let $M$ be an $(f-d)$ cap-set for $X$, let $Y$ be a topologically complete metric space, and let $h: M \rightarrow Y$ be an embedding. Then there is a copy $X^{\prime}$ of $X$ such that $h(M) \subset X^{\prime} \subset Y$ and $h(M)$ is an $(f-d)$ cap-set for $X^{\prime}$.

Proof. Using Theorem 10.1 we can extend $h$ to a homeomorphism $H: X^{\prime \prime} \rightarrow H\left(X^{\prime \prime}\right)$, where $X^{\prime \prime}$ and $H\left(X^{\prime \prime}\right)$ are $G_{\delta}$-sets. Using Lemma 5.8 we find that $X \backslash X^{\prime \prime}=\bigcup_{i=1}^{\infty} F_{i}$, where each $F_{i}$ is a $Z$-set in $X$. Using Theorem 1 of [4] there is a homeomorphism $h$ of $X$ onto $X^{\prime \prime}$. Then $X^{\prime \prime}$ is a copy of $X$ and it is obvious that $M$ is an (f-d) cap-set for $X^{\prime \prime}$. We set $X^{\prime}=H\left(X^{\prime \prime}\right)$ and note that $h(M)$ is an (f-d) cap-set for $X^{\prime}$.

We are now ready for the major step in establishing our open embedding theorems. This will also be used in the next section.

THEOREM 10.2. If $X$ is a $\sigma$ (or $\Sigma$ )-manifold, then there is an F-manifold $Y$ and an embedding $h: X \rightarrow Y$ such that $h(X)$ is an $f$-d cap (or cap)-set for $Y$.

Proof. Let $\left\{U_{i}\right\}_{i=1}^{\infty}$ be an open cover of $X$ such that each $U_{i}$ is homeomorphic to an open subset of $\sigma($ or $\Sigma)$. Without loss of generality assume that $X \subset Z$, where $Z$ is a topologically complete separable metric space. For each $i>0$ let $V_{i}$ be an open subset of $Z$ such that $V_{i} \cap X=U_{i}$ and let $G_{i}$ be an open subset of $s$ such that $G_{i} \cap \sigma\left(\right.$ or $G_{i} \cap \Sigma$ ) is homeomorphic to $U_{i}$. Then $G_{i} \cap \sigma$ (or $G_{i} \cap \Sigma$ ) is an f-d cap (or cap)-set for $G_{i}$, and making use of Lemma 10.1 there is an embedding $h_{i}: G_{i} \rightarrow V_{i}$ such that $U_{i} \subset h_{i}\left(G_{i}\right)$ and $U_{i}$ is an f-d cap (or cap)-set for $h_{i}\left(G_{i}\right)$.

We note that each $h_{i}\left(G_{i}\right)$ is a topologically complete metric space and hence it must be a $G_{\delta}$ in $V_{i}$. Thus for each $i>0$ we have $V_{i} \mid h_{i}\left(G_{i}\right)=\bigcup_{n=1}^{\infty} A_{i}^{n}$, with each $A_{i}^{n}$ closed in $Z$. We also note that for each $i$ and $n, A_{i}^{n} \cap X=\varnothing$. This means that $A_{i}^{n} \cap h_{j}\left(G_{j}\right)$ is a $Z$-set in $h_{j}\left(G_{j}\right)$, for all $n, i$, and $j$. Now put $G_{i}^{\prime}=h_{i}\left(G_{i}\right) \backslash \bigcup_{i=1}^{\infty} \bigcup_{n=1}^{\infty} A_{i}^{n}$, and from Theorem 1 of [4] we know that each $G_{i}^{\prime}$ is homeomorphic to $h_{i}\left(G_{i}\right)$. It is also true that each $U_{i}$ is an f-d cap (or cap)-set for $G_{i}^{\prime}$.

We note that for each $j>0, V_{j} \cap\left(\bigcup_{i=1}^{\infty} G_{i}^{\prime}\right)=G_{j}^{\prime}$. This means that $Y=\bigcup_{i=1}^{\infty} G_{i}^{\prime}$ is an $F$-manifold. Since $X$ has the f-d cap (or cap) locally in $Y$, we find that $X$ is an f-d cap (or cap)-set for $Y$.

We can also prove a corresponding property for $Q$-manifolds. 
COROLlarY 10.1. If $X$ is a $\sigma($ or $\Sigma)$-manifold, then there is a $Q$-manifold $Y$ and an embedding $h: X \rightarrow Y$ such that $h(X)$ is an $f$-d cap (or cap)-set for $Y$.

Proof. Let $Z$ be an $F$-manifold and let $f: X \rightarrow Z$ be an embedding such that $f(X)$ is an $\mathrm{f}$-d cap (or cap)-set for $Z$. Using Theorem 5.1 there is a $Q$-manifold $Y$ and an embedding $g: Z \rightarrow Y$ such that $Y \backslash g(Z)$ is a cap-set for $Y$. Then Theorem 6.3 implies that $g \circ f(X)$ is an $\mathrm{f}$-d cap (or cap)-set for $Y$.

We can easily use Theorem 10.2 to classify $\sigma$ and $\Sigma$-manifolds according to homotopy type.

COROLlaRY 10.2. If $X$ and $Y$ are both $\sigma$-manifolds (or both $\Sigma$-manifolds) and are of the same homotopy type, then they are homeomorphic.

Proof. Using Theorem 10.2 there are $F$-manifolds $X^{\prime}, Y^{\prime}$ and embeddings $f: X \rightarrow X^{\prime}, g: Y \rightarrow Y^{\prime}$ such that $f(X)$ is an $\mathrm{f}$-d cap (or cap)-set for $X^{\prime}$ and $g(Y)$ is an f-d cap (or cap)-set for $Y^{\prime}$. Since $X$ and $Y$ have the same homotopy type, it follows from Corollary 8.2 that $X^{\prime}$ and $Y^{\prime}$ have the same homotopy type. Using Theorem 2.1 we have $X^{\prime}$ homeomorphic to $Y^{\prime}$. Then Theorem 6.1 implies that $f(X)$ is homeomorphic to $g(Y)$.

We can also use these techniques to sharpen Corollary 8.1.

Corollary 10.3. Let $X$ be a $\sigma$-manifold and let $Y$ be a $\Sigma$-manifold of the same homotopy type. Then $X \times I^{\infty}$ and $Y$ are homeomorphic.

Proof. Using the techniques of the proof of Corollary 10.2 there is an $F$-manifold $Z$ and embeddings $f: X \rightarrow Z, g: Y \rightarrow Z$ such that $f(X)$ is an f-d cap-set for $Z$ and $g(Y)$ is a cap-set for $Z$. Then Corollary 8.1 implies that $f(X) \times I^{\infty}$ and $g(Y)$ are homeomorphic.

Finally we sharpen Theorem 8.2.

Corollary 10.4. Let $X$ be a $\sigma$ (or $\Sigma$ )-manifold. Then there is a locally-finite simplicial complex $K$ such that $X$ is homeomorphic to $|K| \times \sigma($ or $|K| \times \Sigma)$.

Proof. This is an immediate application of Theorem 10.2.

We now use Theorem 10.2 to establish open embedding theorems for $\sigma$ and $\Sigma$ manifolds.

THEOREM 10.3. Let $X$ be a $\sigma($ or $\Sigma)$-manifold. Then $X$ can be embedded as an open subset of $\sigma($ or $\Sigma)$.

Proof. Using Theorem 10.2 there is an embedding $h: X \rightarrow Y$, where $Y$ is an $F$ manifold and $h(X)$ is an $\mathrm{f}$-d cap (or cap)-set for $Y$. Using Henderson's open embedding theorem there is an open embedding $g: Y \rightarrow s$. Then $g \circ h(X)$ is an f-d cap (or cap)-set for $g(Y)$, which is an open subset of $s$. Also $g(Y) \cap \sigma$ (or $g(Y) \cap \Sigma$ ) is an open subset of $\sigma$ (or $\Sigma$ ) and has the f-d cap (or cap) in $g(Y)$. By Theorem 6.1 we have $g \circ h(X)$ and $g(Y) \cap \sigma($ or $g(Y) \cap \Sigma)$ homeomorphic, which is what we wished to prove. 
11. Extending homeomorphisms in $\sigma$ and $\Sigma$-manifolds. The main result of this section is Theorem 11.1 in which we establish a homeomorphism extension theorem for $\sigma$ and $\Sigma$-manifolds. Our strategy is to use techniques similar to those used in $\S 10$ to transfer the problem to an $F$-manifold and then apply Theorem 2.24.

We will need a number of preliminary results. The first two of these follow routinely from a close examination of Lavrent'ev's theorem on p. 335 of [16].

LeMma 11.1 Let $X$ be a topological space, let $Y$ be a topologically complete metric space, and let $f: A \rightarrow Y$ be a continuous function, where $A \subset X$. Then there is $a$ $G_{\delta}$-subset $B$ of $X$ such that $A \subset B \subset \bar{A}$ and an extension of $f$ to a continuous function $g: B \rightarrow Y$.

LEMMA 11.2. Let $X$ and $Y$ be topologically complete metric spaces, let $A$ be a subset of $X$, and let $f: A \rightarrow Y$ be an embedding. If $B$ is $a G_{\delta}$-subset of $X$ and $g: B \rightarrow Y$ is a continuous extension of $f$, then there is a $G_{\delta}$-subset $B^{\prime}$ of $X$ such that $A \subset B^{\prime} \subset \bar{A}$ and $g \mid B^{\prime}$ is an embedding.

The proof of our next lemma is elementary.

LEMMA 11.3. Let $X$ be a metric space and let $Y$ be a compact metric space. Then the projection $\pi_{X}$ is a closed map.

We now combine these results to show that we can extend homotopies.

LEMMA 11.4. Let $X$ be a topologically complete metric space and let $A$ be a subset of $X$. Let $h: A \times I \rightarrow Y$, where $Y$ is a topologically complete metric space, be a homotopy such that $h \mid A \times\{0\}$ and $h \mid A \times\{1\}$ are embeddings. Then there is a $G_{\delta^{-}}$ subset $B$ of $X$ such that $A \subset B \subset \bar{A}$ and an extension of $h$ to a homotopy $H: B \times I \rightarrow Y$ such that $H \mid B \times\{0\}$ and $H \mid B \times\{1\}$ are embeddings.

Proof. Using Lemma 11.1 there is a $G_{\delta}$-subset $C$ of $X \times I$ such that $A \times I \subset C$ $\subset \mathrm{Cl}(A \times I)$ (closure taken in $X \times I)$ and an extension of $h$ to a continuous function $f: C \rightarrow Y$. Using Lemma 11.3 we find that $\pi_{X}((X \times I) \mid C)=D$ is an $F_{\sigma}$-subset of $X$. Then $B_{1}=X \backslash D$ is a $G_{\delta}$-subset of $X$ such that $B_{1} \times I \subset C$, which means that $g=f \mid B_{1} \times I$ is an extension of $h$.

Applying Lemma 11.2 to $g \mid B_{1} \times\{0\}$ and $g \mid B_{1} \times\{1\}$ we find that there are $G_{\delta^{-}}$ subsets $B_{2}$ and $B_{3}$ of $X$ such that $A \subset B_{2} \subset B_{1}, A \subset B_{3} \subset B_{1}, g \mid B_{2} \times\{0\}$ is an embedding, and $g \mid B_{3} \times\{0\}$ is an embedding. Then put $B=B_{2} \cap B_{3}$ and $H=g \mid B \times I$ to fulfill the conditions of the lemma.

If we examine the proof of Lemma 11.4 we can easily sharpen it.

COROLLARY 11.1. Using the notation of Lemma 11.4 let $\mathscr{U}$ be an open cover of $Y$ such that for each $x \in A, h(\{x\} \times I)$ is contained in some member of $\mathscr{U}$. Then we can construct $H$ so that for each $x \in B, H(\{x\} \times I)$ is contained in some member of St $(\mathscr{U})$.

We are now ready to prove our main result. 
THeOREM 11.1. Let $X$ be a $\Sigma$ (or $\sigma)$-manifold, let $\mathscr{U}$ be an open cover of $X$, let $A$ be a separable metric space, and let $f: A \times I \rightarrow X$ be a homotopy such that $f \mid A \times\{0\}$ and $f \mid A \times\{1\}$ are embeddings, $f(A \times\{0\})$ and $f(A \times\{1\})$ are Z-sets in $X$ and for each $x \in A, f(\{x\} \times I)$ is contained in some member of $\mathscr{U}$. Then the induced homeomorphism of $f(A \times\{0\})$ onto $f(A \times\{1\})$ can be extended to a homeomorphism of $X$ onto itself which is limited by $\mathrm{St}^{28}(\mathscr{U})$.

Proof. Without loss of generality assume that $X \subset Y$, where $Y$ is an $F$-manifold and $X$ is an (f-d) cap-set for $Y$. Moreover we can assume that for each $U \in \mathscr{U}$, there is an open subset $V_{U}$ of $Y$ such that $V_{U} \cap X=U$ and $Y=\bigcup\left\{V_{U} \mid U \in \mathscr{U}\right\}$.

Let $Z$ be a topologically complete metric space containing $A$ and use Lemma 11.4 to get a $G_{\delta}$-subset $B$ of $Z$ such that $A \subset B \subset \bar{A}$ and an extension of $f$ to a homotopy $f_{1}: B \times I \rightarrow Y$ such that $f_{1} \mid B \times\{0\}$ and $f_{1} \mid B \times\{1\}$ are embeddings. Using Corollary 11.1 we can additionally assume that for each $x \in B, f_{1}(\{x\} \times I)$ is contained in some member of St $(\mathscr{V})$, where $\mathscr{V}=\left\{V_{U} \mid U \in \mathscr{U}\right\}$.

Now put $F_{1}=\mathrm{Cl}(f(A \times\{0\}))$ and $F_{2}=\mathrm{Cl}(f(A \times\{1\}))$, where the closure is taken in $Y$, and note that Lemma 5.10 implies that $F_{1}$ and $F_{2}$ are $Z$-sets in $Y$.

Since $Y \times B\left(I^{\infty}\right)$ is a cap-set for $Y \times I^{\infty}$, there is a homeomorphism $h_{1}: Y \rightarrow Y \times I^{\infty}$ such that $h_{1}(X)=Y \times B\left(I^{\infty}\right)$. Using Theorem 7.2 there is a homeomorphism $h_{2}: Y \times I^{\infty} \rightarrow Y \times I^{\infty}$ such that $\pi_{I}^{\infty} \circ h_{2} \circ h_{1}\left(F_{1} \cup F_{2}\right)$ has infinite deficiency and $h_{2}\left(Y \times B\left(I^{\infty}\right)\right)=Y \times B\left(I^{\infty}\right)$. It is obvious that by adjusting one of the coordinates in which $\pi_{I}^{\infty} \circ h_{2} \circ h_{1}\left(F_{1} \cup F_{2}\right)$ is deficient we can get a homeomorphism $h_{3}: Y \times I^{\infty} \rightarrow Y \times I^{\infty}$ such that $h_{3} \circ h_{2} \circ h_{1}\left(F_{1} \cup F_{2}\right) \cap h_{2} \circ h_{1}\left(F_{1} \cup F_{2}\right)=\varnothing$, $h_{3}\left(Y \times B\left(I^{\infty}\right)\right)=Y \times B\left(I^{\infty}\right)$, and $h_{3}$ is limited by $h_{2} \circ h_{1}(\mathscr{V})$. Then $h=\left(h_{2} \circ h_{1}\right)^{-1}$ $\circ h_{3} \circ h_{2} \circ h_{1}$ is a homeomorphism of $Y$ onto itself such that $h(X)=X, h\left(F_{2}\right) \cap F_{1}=\varnothing$, and $h$ is limited by $\mathscr{V}$.

Using the construction of $h_{2}$ we can easily get a homotopy $F: Y \times I \rightarrow Y$ such that for each $x \in Y, F(x, 0)=x, F(x, 1)=h(x)$, and $F(\{x\} \times I)$ is contained in some member of $\mathscr{V}$. We can clearly use $F$ to modify $f_{1}$ to a homotopy $f_{2}: B \times I \rightarrow Y$ such that $f_{2}\left|B \times\{0\}=f_{1}\right| B \times\{0\}, f_{2}\left|B \times\{1\}=h \circ f_{1}\right| B \times\{1\}$, and for each $x \in B, f_{2}(\{x\} \times I)$ is contained in some member of $\mathrm{St}^{2}(\mathscr{V})$.

Now let $g_{1}$ be a homeomorphism of $Y$ onto $Y \times I^{\infty}$ such that $\pi_{I} \infty \circ g_{1}\left(F_{1} \cup h\left(F_{2}\right)\right)$ has infinite deficiency. Without loss of generality we may assume that $\tau_{1} \circ \pi_{I} \circ g_{1}\left(F_{1} \cup h\left(F_{2}\right)\right)=\{0\}$. Now apply Theorem 2.24 to obtain a homeomorphism $g_{2}$ of $Y \times I^{\infty}$ onto itself such that $\tau_{1} \circ \pi_{I}^{\infty} \circ g_{2} \circ g_{1}\left(F_{1} \cup h\left(F_{2}\right)\right)$ $=\{-1\}$.

Using this fact we can easily get a homotopy $G: Y \times I \rightarrow Y$ such that for $x \in Y$ and $t \in(0,1), G(x, 0)=G(x, 1)=x, G(x, t) \notin F_{1} \cup h\left(F_{2}\right)$, and $G(\{x\} \times I)$ is contained in some member of $\mathscr{V}$. We can obviously use $G$ to modify $f_{2}$ to a homotopy $f_{3}: B \times I \rightarrow Y$ such that $f_{3}\left|B \times\{0\}=f_{1}\right| B \times\{0\}, f_{3}\left|B \times\{1\}=h \circ f_{1}\right| B \times\{1\}, f_{3}(B \times(0,1))$ $\cap\left(F_{1} \cup h\left(F_{2}\right)\right)=\varnothing$, and for each $x \in B, f_{3}(\{x\} \times I)$ is contained in some member of $\mathrm{St}^{3}(\mathscr{V})$. 
Put $C_{1}=f_{3}(B \times\{0\})$, which is a $G_{\delta}$-subset of $F_{1}$, and $C_{2}=f_{3}(B \times\{1\})$, which is a $G_{\delta}$-subset of $h\left(F_{2}\right)$. Let $F_{1} \backslash C_{1}=\bigcup_{n=1}^{\infty} A_{n}$ and $h\left(F_{2}\right) \backslash C_{2}=\bigcup_{n=1}^{\infty} B_{n}$, where each $A_{n}$ and each $B_{n}$ is a closed subset of $Y$. It is then clear that $\left(\bigcup_{n=1}^{\infty} A_{n}\right) \cap\left(\bigcup_{n=1}^{\infty} B_{n}\right)=\varnothing$ and for each $n>0, A_{n}, B_{n}$ are $Z$-sets in $Y$.

Let $Y^{\prime}=Y \backslash \bigcup_{n=1}^{\infty}\left(A_{n} \cup B_{n}\right)$, which is homeomorphic to $Y$ by Theorem I of [4], and let $\mathscr{V}^{\prime}=\left\{V \cap Y^{\prime} \mid V \in \mathscr{V}\right\}$. We note that $C_{1}$ and $C_{2}$ are $Z$-sets in $Y^{\prime}$ and $f_{3}(B \times I) \subset Y^{\prime}$. If we let $\varphi$ be the homeomorphism of $C_{1}$ onto $C_{2}$ induced by $f_{3}$, then there is a homotopy $\Phi: C_{1} \times I \rightarrow Y$ such that for each $x \in C_{1}, \Phi(x, 0)=x, \Phi(x, 1)$ $=\varphi(x)$, and $\Phi(\{x\} \times I)$ is contained in some member of $\mathrm{St}^{3}\left(\mathscr{V}^{\prime}\right)$.

Define $\varphi_{1}: C_{1} \cup C_{2} \rightarrow C_{1} \cup C_{2}$ by $\varphi_{1} \mid C_{1}=\varphi$ and $\varphi_{1} \mid C_{2}=\varphi^{-1}$. Clearly there is a homotopy $\Phi_{1}:\left(C_{1} \cup C_{2}\right) \times I \rightarrow Y$ such that for each $x \in C_{1} \cup C_{2}, \Phi_{1}(x, 0)=x$, $\Phi_{1}(x, 1)=\varphi_{1}(x)$, and $\Phi_{1}(\{x\} \times I)$ is contained in some member of $\mathrm{St}^{3}\left(\mathscr{V}^{\prime}\right)$. Moreover, we note that $\varphi_{1}\left(\left(C_{1} \cup C_{2}\right) \cap X\right)=\left(C_{1} \cup C_{2}\right) \cap X$.

Since $X \mid C_{1} \cup C_{2}$ is an (f-d) cap-set for $Y^{\prime}$, there is a homeomorphism $\psi_{1}$ of $Y^{\prime}$ onto itself such that $\psi_{1}\left(X \backslash\left(C_{1} \cup C_{2}\right)\right)=X$ and $\psi_{1}$ is limited by $\mathscr{V}^{\prime}$. Using Theorem 2.24 there is a homeomorphism $\psi_{2}$ of $Y^{\prime}$ onto itself which extends the homeomorphism $\psi_{1} \circ \varphi_{1} \circ \psi_{1}^{-1}: \psi_{1}\left(C_{1} \cup C_{2}\right) \rightarrow \psi_{1}\left(C_{1} \cup C_{2}\right)$ and is limited by $\operatorname{St}^{24}\left(\mathscr{V}^{\prime}\right)$. Using Theorem 6.1 there is a homeomorphism $\psi_{3}$ of $Y^{\prime}$ onto itself such that $\psi_{3}\left(\psi_{2}(X)\right)=X, \psi_{3} \mid \psi_{1}\left(C_{1} \cup C_{2}\right)=\mathrm{id}$, and $\psi_{3}$ is limited by $\mathscr{V}^{\prime}$. Then $\psi=\psi_{1}^{-1} \circ \psi_{3}$ $\circ \psi_{2} \circ \psi_{1}$ is a homeomorphism of $Y^{\prime}$ onto itself such that $\psi(X)=X, \psi$ extends $\varphi$, and $\psi$ is limited by $\mathrm{St}^{27}\left(\mathscr{V}^{\prime}\right)$.

We note now that $g=\left(h^{-1} \mid X\right) \circ(\psi \mid X)$ is a homeomorphism of $X$ onto itself which fulfills our requirements.

By examining the proof of Theorem 11.1 we can state a stronger result.

COROLlary 11.2. Let the hypotheses be as in Theorem 11.1. Then there is an Fmanifold $Z$ containing $X$ and an open cover $\mathscr{V}$ of $Z$ such that $\{V \cap X \mid V \in \mathscr{V}\}=\mathscr{U}$ and for which the induced homeomorphism of $f(A \times\{0\})$ onto $f(A \times\{1\})$ can be extended to a homeomorphism of $Z$ onto itself which takes $X$ onto itself and is limited by $\mathrm{St}^{28}(\mathscr{V})$.

If we apply Theorem 11.1 to $\sigma$ or $\Sigma$ we can easily obtain the following homeomorphism extension theorem, where the homotopy takes place along straight line segments joining each point to its image.

Corollary 11.3. Let $X=\sigma$ or $\Sigma$, let $K_{1}$ and $K_{2}$ be Z-sets in $X$, let $\varepsilon>0$ be given, and let $h$ be a homeomorphism of $K_{1}$ onto $K_{2}$ such that $d(h, \mathrm{id})<\varepsilon$. Then $h$ can be extended to a homeomorphism $H$ of $X$ onto itself such that $d(H, \mathrm{id})<29 \varepsilon$.

We remark that by exercising a bit more care the estimate $\operatorname{St}^{28}(\mathscr{U})$ in Theorem 11.1 can be improved upon, however it does not seem worth the effort. In regard to Corollary 11.3 it is possible to give a proof independent of Theorem 11.1 and obtain the estimate $2 \varepsilon$, where Theorem 11.1 would be replaced by the homeomorphism extension theorem announced by Barit [11]. 


\section{REFERENCES}

1. R. D. Anderson, Hilbert space is homeomorphic to the countable infinite product of lines, Bull. Amer. Math. Soc. 72 (1966), 515-519. MR 32 \#8298.

2. - Topological properties of the Hilbert cube and the infinite product of open intervals, Trans. Amer. Math. Soc. 126 (1967), 200-216. MR 34 \#5045.

3. - On topological infinite deficiency, Michigan Math. J. 14 (1967), 365-383. MR 35 \#4893.

4. - Strongly negligible sets in Fréchet manifolds, Bull. Amer. Math. Soc. 75 (1969), 64-67. MR 38 \#6634.

5. - A characterization of apparent boundaries of the Hilbert cube, Notices Amer. Math. Soc. 16 (1969), 429. Abstract \#697-G17.

6. - On sigma-compact subsets of infinite-dimensional spaces, Trans. Amer. Math. Soc. (submitted).

7. R. D. Anderson and R. H. Bing, A complete elementary proof that Hilbert space is homeomorphic to the countable infinite product of lines, Bull. Amer. Math. Soc. 74 (1968), 771-792. MR 37 \#5847.

8. R. D. Anderson and John D. McCharen, On extending homeomorphisms to Fréchet manifolds, Proc. Amer. Math. Soc. 25 (1970), 283-289.

9. R. D. Anderson and R. Schori, Factors of infinite-dimensional manifolds, Trans. Amer. Math. Soc. 142 (1969), 315-330.

10. R. D. Anderson, David W. Henderson and James E. West, Negligible subsets of infinitedimensional manifolds, Compositio Math. 21 (1969), 143-150.

11. William Barit, Small extensions of small homeomorphisms, Notices Amer. Math. Soc. 16 (1969), 295. Abstract \#663-715.

12. C. Bessaga and A. Pelczyński, Estimated extension theorem, homogeneous collections and skeletons, and their applications to topological classifications of linear metric spaces and convex sets, Fund. Math. (submitted).

13. T. A. Chapman, Infinite deficiency in Fréchet manifolds, Trans. Amer. Math. Soc. 148 (1970), 137-146.

14. David W. Henderson, Infinite-dimensional manifolds are open subsets of Hilbert space, Bull. Amer. Math. Soc. 75 (1969), 759-762.

15. W. Hurewicz and H. Wallman, Dimension theory, Princeton Math. Series, vol. 4, Princeton Univ. Press, Princeton, N. J., 1941. MR 3, 312.

16. K. Kuratowski, Topologie. Vol. 1: Espaces métrisables, espaces complets, 2nd ed., Monografie Mat., Tom 20, PWN, Warsaw, 1948. MR 10, 389.

17. H. Torunczyk, Skeletonized sets in complete metric spaces and homeomorphisms of the Hilbert cube, Bull. Acad. Polon. Sci. Cl. III 18 (1970), 119-126.

18. James E. West, Infinite products which are Hilbert cubes, Trans. Amer. Math. Soc. 150 (1970), 1-25.

Louisiana State University, Baton Rouge, LoUisiana 70803 